Discrete Comput Geom 33:565-591 (2005)

DOI: $10.1007 / \mathrm{s} 00454-005-1168-0$

\title{
An Improved Bound on the One-Sided Minimum Crossing Number in Two-Layered Drawings*
}

\author{
Hiroshi Nagamochi \\ Department of Applied Mathematics and Physics, Kyoto University, \\ Kyoto 606-8501, Japan \\ nag@amp.i.kyoto-u.ac.jp
}

\begin{abstract}
Given a bipartite graph $G=(V, W, E)$, a two-layered drawing consists of placing nodes in the first node set $V$ on a straight line $L_{1}$ and placing nodes in the second node set $W$ on a parallel line $L_{2}$. The one-sided crossing minimization problem asks one to find an ordering of nodes in $V$ to be placed on $L_{1}$ so that the number of arc crossings is minimized. In this paper we use a 1.4664-approximation algorithm for this problem. This improves the previously best bound 3 due to P. Eades and N. C. Wormald [Edge crossing in drawing bipartite graphs, Algorithmica 11 (1994), 379-403].
\end{abstract}

\section{Introduction}

Given a bipartite graph $G=(V, W, E)$, a two-layered drawing consists of placing nodes in the first node set $V$ on a straight line $L_{1}$ and placing nodes in the second node set $W$ on a parallel line $L_{2}$. The problem of minimizing the number of crossings between arcs in a two-layered drawing was first introduced by Harary and Schwenk [6], [7]. The one-sided crossing minimization problem asks one to find an ordering of nodes in $V$ to be placed on $L_{1}$ so that the number of arc crossings is minimized (while the ordering of the nodes in $W$ on $L_{2}$ is given and fixed).

The problem has many applications such as VLSI layouts [14] and hierarchical drawings [1], [15]. However, the two-sided and one-sided problems are shown to be NP-hard by Garey and Johnson [5] and by Eades and Wormald [4], respectively. Muñoz et al. [11] have proven that the one-sided problem remains NP-hard even for sparse graphs

* A preliminary version of this paper appeared in Proceedings of the 11th International Symposium on Graph Drawing (GD2003), Lecture Notes in Computer Science, Vol. 2912. This research was partially supported by a Scientific Grant in Aid from the Ministry of Education, Culture, Sports, Science and Technology of Japan. 
such as forests of 4-stars. Dujmović and Whitesides [3] have given an $O\left(\varphi^{k} \cdot n^{2}\right)$ time algorithm to the one-sided problem, where $k$ is the number of crossings to be checked, $n=|V|+|W|$ and $\varphi=(1+\sqrt{5}) / 2$, thus showing that the problem is Fixed Parameter Tractable. Recently Dujmović et al. [2] gave an $O\left(1.4656^{k}+k|V|^{2}\right)$ time algorithm for this problem.

In this paper we consider the one-sided problem. For this problem, there are several heuristics that deliver theoretically or empirically good solutions. The so-called barycentre heuristic finds an $O(\sqrt{n})$-approximation solution or a $(d-1)$-approximation solution, where $d$ is the maximum degree of nodes in the free side $V$ (see [9] for the analysis). Eades and Wormald [4] proposed a simple and theoretically better heuristic, the median heuristic, which constructs an ordering of $V$ by aligning each node $v \in V$ on $L_{1}$ with respect to the position in $L_{2}$ of the median neighbour of $v$ (breaking ties by an adequate rule). They prove that the median heuristic delivers a 3 -approximation solution, and its performance guarantee approaches to 1 if graphs become dense. Yamaguchi and Sugimoto [16] gave a 2-approximation algorithm if $d \leq 4$. All the known performance guarantees of these heuristics are based on a conventional lower bound that is obtained by summing up $\min \left\{c_{u v}, c_{v u}\right\}$ over all node pairs $u, v \in V$, where $c_{u v}$ denotes the number of crossings generated by arcs incident to $u$ and $v$ when $u$ precedes $v$ in an ordering. An extensive computational experiment of several heuristics including the above two has been conducted by Jünger and Mutzel [8] and by Mäkinen [10]. Jünger and Mutzel [8] reported that most of the heuristics gave good solutions whose crossing numbers are nearly equal to the lower bound. However, the theoretically best estimation to the gap between the optimum and the lower bound is 3 due to the heuristic by Eades and Wormald [4].

In this paper we prove that, for the one-sided problem, there always exists a solution whose crossing number is at most 1.4664 times the lower bound. Our argument is based on a probabilistic analysis, which provides a polynomial randomized algorithm that delivers a solution whose average number of crossings is at most 1.4664 times the optimum. The algorithm can be evolved from the median heuristic. A first step might be to modify the median heuristic as follows: instead of aligning a node $u$ with its median neighbour, it can be aligned with a neighbour randomly chosen to be in some interval of neighbours, say between the first quartile and the median, or between the $\left\lceil s d_{u}\right\rceil$ th neighbour and the $\left\lceil t d_{u}\right\rceil$ th neighbour for some specified constants $0<s \leq t \leq 1$, where $d_{u}$ denotes the degree of $u$. The algorithm in this paper is even more sophisticated than this, and first chooses the appropriate interval at random (as well as some care taken in the case of ties).

The paper is organized as follows. In Section 2 we introduce basic definitions on twolayered drawing and a geometric representation for crossing numbers $c_{u v}$ and $c_{v u}$ for two nodes $u, v \in V$. In Section 3 we propose a probabilistic algorithm for determining a two-layered drawing and show some basic properties for analysing the algorithm. In Section 4 we show that the algorithm can deliver a solution whose crossing number is at most 1.4664 times the lower bound. In Section 5 we, however, show that our approach cannot prove that the gap between the optimum and the lower bound is less than 1.4414. In Section 6 we discuss deterministic approximation algorithms based on our probabilistic algorithm proposed in Section 3. In Section 7 we make some concluding remarks. 


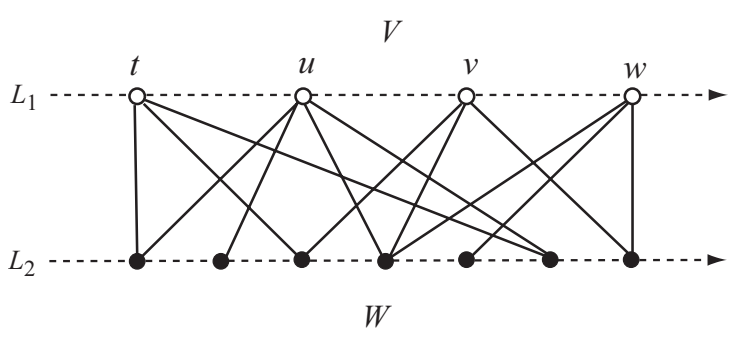

(a)

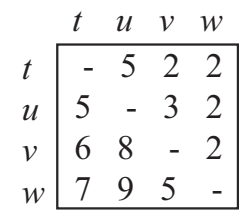

(b)

Fig. 1. (a) A two-layered drawing of a bipartite graph. (b) Crossing numbers for each pair of nodes in the top layer.

\section{Preliminaries}

Let $G=(V, W, E)$ be a bipartite graph with a partition $V$ and $W$ of a node set. Assume that $G$ has no isolated node. Let $\pi$ denote a permutation of $\{1,2, \ldots,|V|\}$ and $\sigma$ denote a permutation of $\{1,2, \ldots,|W|\}$. A pair of $\pi$ and $\sigma$ defines a two-layered drawing of $G$ in the plane in such a way that, for two parallel horizontal lines $L_{1}$ and $L_{2}$, the nodes in $V$ (resp., in $W$ ) are arranged on $L_{1}$ (resp., $L_{2}$ ) according to $\pi$ (resp., $\sigma$ ) and each arc is depicted by a straight line segment joining the endnodes, where the directions for traversing $L_{1}$ and $L_{2}$ are taken as the same (see Fig. 1(a)). For any choice of coordinates of points for nodes in $V \cup W$ in a two-layered drawing of $G$ defined by $(\pi, \sigma)$, two arcs $(v, w),\left(v^{\prime}, w^{\prime}\right) \in E$ intersect properly (or create a crossing) if and only if $\pi(v)<\pi\left(v^{\prime}\right)$ and $\sigma(w)>\sigma\left(w^{\prime}\right)$ (or $\left.\pi(v)>\pi\left(v^{\prime}\right), \sigma(w)<\sigma\left(w^{\prime}\right)\right)$. So we simply call a pair $(\pi, \sigma)$ a two-layered drawing of $G$. In this paper we consider the following problem.

One-Sided Crossing Minimization. Given a bipartite graph $G=(V, W, E)$ and a permutation $\sigma$ on $W$, find a permutation $\pi$ on $V$ that minimizes the number of crossings in a two-layered drawing $(\pi, \sigma)$ of $G$.

Since the permutation $\sigma$ on $W=\{1,2, \ldots,|W|\}$ is fixed, we assume throughout the paper that $\sigma(i)=i$ for all $i \in W$. For each node $u$ in $G$, let $\Gamma(u)$ denote the set of nodes adjacent to $u$, and let $d_{u}=|\Gamma(u)|$. For two nodes $u, v \in V$, let $\Delta_{u v}=|\Gamma(u) \cap \Gamma(v)|$. The crossing number $c_{u v}$ for an ordered pair of two nodes $u, v \in V$ is the number of crossings generated by an arc incident to $u$ and an arc incident to $v$ when $\pi(u)<\pi(v)$ holds in a two-layered drawing $(\pi, \sigma)$. (Figure 1(b) shows the crossing numbers in the graph in Fig. 1(a).) It is a simple matter to see that

$$
\begin{gathered}
d_{u} d_{v}=c_{u v}+c_{v u}+\Delta_{u v}, \\
\min \left\{c_{u v}, c_{v u}\right\} \geq \frac{\Delta_{u v}\left(\Delta_{u v}-1\right)}{2} .
\end{gathered}
$$

For a permutation $\pi$ on $V$, let

$$
\operatorname{cross}(u, v ; \pi):= \begin{cases}c_{u v} & \text { if } \pi(u)<\pi(v), \\ c_{v u} & \text { otherwise. }\end{cases}
$$




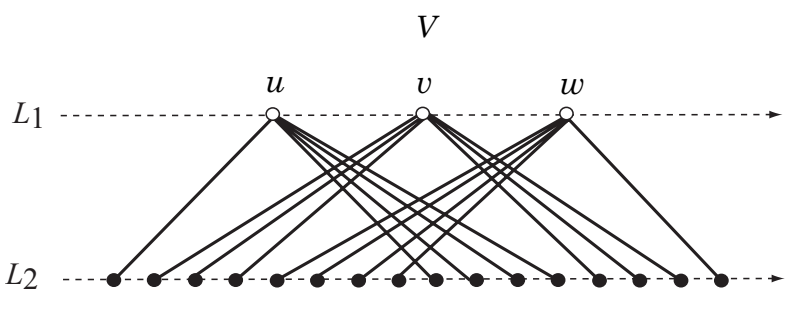

W

(a)

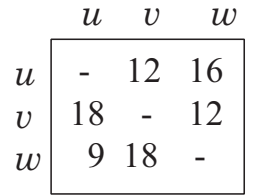

(b)

Fig. 2. (a) A two-layered drawing of a bipartite graph. (b) Crossing numbers for each pair of nodes in the top layer.

Define

$$
\operatorname{cross}(\pi):=\sum_{u, v \in V: \pi(u)<\pi(v)} c_{u v}=\sum_{u, v \in V} \operatorname{cross}(u, v ; \pi) .
$$

The optimum of the problem is denoted by opt $=\min \{\operatorname{cross}(\pi) \mid$ permutation $\pi$ on $V\}$. For $L B=\sum_{u, v \in V} \min \left\{c_{u v}, c_{v u}\right\}$, it holds that

$$
o p t \geq L B \text {. }
$$

Figure 2 shows an example such that $o p t=39$ and $L B=33$. Hence the maximum ratio $L B /$ opt over all bipartite graphs is at least $13 / 11 \simeq 1.1818$. In this paper we prove the next result.

Theorem 1. For a bipartite graph $G=(V, W, E)$ with a permutation $\sigma$ on $W$, there exists a permutation $\pi$ on $V$ such that $\operatorname{cross}(\pi) \leq 1.4664 L B$.

Note that the one-sided crossing minimization is a purely combinatorial problem in the sense that the number of crossings is determined by a permutation $\pi$, not by the actual positions of nodes in the layers. However, in this paper we convert the problem into a geometric problem to derive Theorem 1 . For this, we first introduce a geometric representation that illustrates how two sets $\Gamma(u)$ and $\Gamma(v)$ determine crossing numbers $c_{u v}$ and $c_{v u}$ in a bipartite graph $G$. The rectangles that we treat here are axis-parallel in the $x y$-coordinate, and they are denoted by the coordinates of the lower-left corner and the upper-right corner, where the $x$-coordinate increases in the right direction and the $y$-coordinate increases in the upward direction. For example, $\left[(0,0),\left(\frac{1}{2}, 1\right)\right]$ represents the square with four corners $(0,0),(0,1),\left(\frac{1}{2}, 0\right)$ and $\left(\frac{1}{2}, 1\right)$.

Let $S$ denote the square $[(0,0),(1,1)]$. For a connected region $R$ in $S$, we may use $R$ to denote the sets of points in the region $R$, and let $a(R)$ denote the area of $R$. For two points $b, b^{\prime} \in S$, a line segment connecting $b$ and $b^{\prime}$ is denoted by $b b^{\prime}$. A part of the boundary of a region $R$ may be called an $e d g e$ if it is a line segment. For a line segment (or an edge) $e$, its length is denoted by $\ell(e)$. We say that edge $e$ overlaps with another edge $e^{\prime}$ if the intersection of $e$ and $e^{\prime}$ is a line segment of a positive length. 


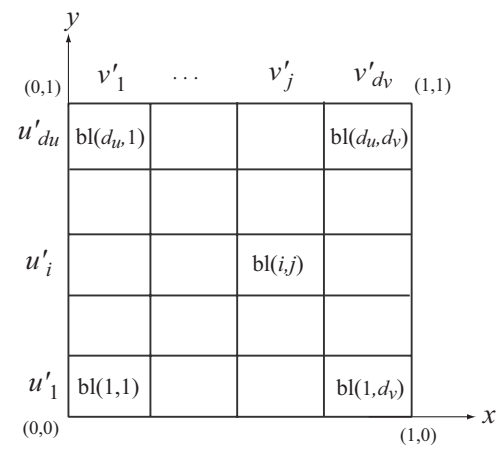

Fig. 3. Illustration for blocks in a $\left(d_{u}, d_{v}\right)$-sliced square $S$.

A path $P$ between points $(0,0)$ and $(1,1)$ in $S$ is called monotone if neither of the $x$ and $y$-coordinates of the point on $P$ decreases when we traverse points on $P$ from $(0,0)$ to $(1,1)$ (in general a monotone path is not necessarily piecewise linear).

For two integers $d, d^{\prime} \geq 1$, the square $S=[(0,0),(1,1)]$ is called $\left(d, d^{\prime}\right)$-sliced if it is sliced by $(d-1)$ horizontal line segments and $\left(d^{\prime}-1\right)$ vertical segments so that these line segments give rise to $d \times d^{\prime}$ congruent rectangles (see Fig. 3). Each such rectangle is called a block, which has four edges.

We represent the positions of nodes in $\Gamma(u)$ and $\Gamma(v)$ in the permutation $\sigma$ by using the unit square $S$ in the $x y$-coordinate. Let $\Gamma(u)=\left\{u_{1}^{\prime}, u_{2}^{\prime}, \ldots, u_{d_{u}}^{\prime}\right\}$ and $\Gamma(v)=$ $\left\{v_{1}^{\prime}, v_{2}^{\prime}, \ldots, v_{d_{v}}^{\prime}\right\}$. For an ordered pair $(u, v)$ of nodes in $V$, we consider $d_{u} d_{v}$ blocks in the $\left(d_{u}, d_{v}\right)$-sliced square $S$. We denote these blocks by

$$
\mathrm{bl}(i, j)=\left[\left(\frac{j-1}{d_{v}}, \frac{i-1}{d_{u}}\right),\left(\frac{j}{d_{v}}, \frac{i}{d_{u}}\right)\right], \quad 1 \leq i \leq d_{u} \quad \text { and } \quad 1 \leq j \leq d_{v}
$$

(see Fig. 3). We let bl $(i, j)$ correspond to a pair of $\operatorname{arcs}\left(u, u_{i}^{\prime}\right)$ and $\left(v, v_{j}^{\prime}\right)$. Note that $\operatorname{arcs}\left(u, u_{i}^{\prime}\right)$ and $\left(v, v_{j}^{\prime}\right)$ create a crossing in a permutation $\pi$ with $\pi(u)<\pi(v)$ or $\pi(u)>\pi(v)$ if $u_{i}^{\prime} \neq v_{j}^{\prime}$, but generate no crossing in any permutation $\pi$ otherwise. We call a block bl $(i, j)$ with $u_{i}^{\prime} \neq v_{j}^{\prime}$ an $u p$-block if arcs $\left(u, u_{i}^{\prime}\right)$ and $\left(v, v_{j}^{\prime}\right)$ create a crossing in a permutation $\pi$ with $\pi(u)<\pi(v)$ and a down-block otherwise. We call a block bl $(i, j)$ with $u_{i}^{\prime}=v_{j}^{\prime}$ a neutral-block. Observe that the number of up-blocks (resp., down-blocks and neutral-blocks) is equal to $c_{u v}$ (resp., $c_{v u}$ and $\Delta_{u v}=\Delta_{v u}$ ). Here we partition the set of these blocks into two groups $U P$ and $D W N$ as follows (where a neutral-block may be split into two half-blocks in the partitioning).

Definition 2. For each node $u \in V$, where $\Gamma(u)=\left\{w_{1}, w_{2}, \ldots, w_{d_{u}}\right\} \subseteq\{1,2, \ldots,|W|\}$ $\left(w_{1}<w_{2}<\cdots<w_{d_{u}}\right)$, we define the median index $\mu(u)$ of its neighbours by

$$
\mu(u):= \begin{cases}w_{\left(d_{u}+1\right) / 2} & \text { if } d_{u} \text { is odd } \\ \frac{1}{2}\left(w_{\left(d_{u}\right) / 2}+w_{\left(d_{u}\right) / 2+1}\right) & \text { if } d_{u} \text { is even. }\end{cases}
$$




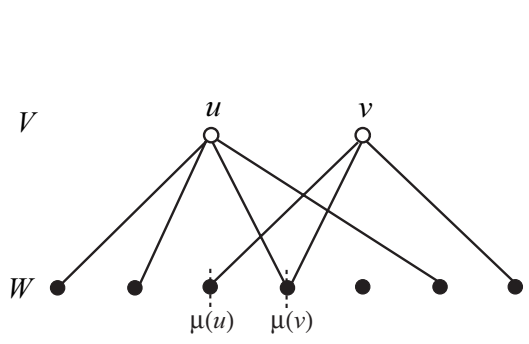

(a)

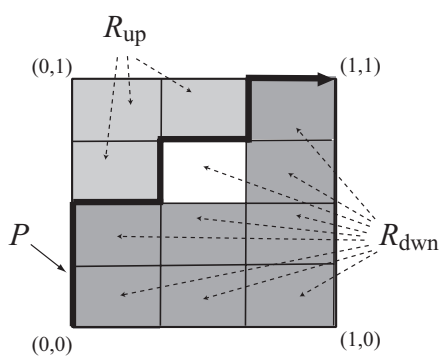

(b)

Fig. 4. (a) Two nodes $u$ and $v$ in the top layer, where $c_{u v}=3$ and $c_{v u}=8$. (b) $\mathrm{A}(u, v)$-path $P$ of a $(4,3)$-sliced square $S$ in the case of (i).

(i) If $\mu(u)<\mu(v)$, then let $U P$ be the set of all up-blocks, and $D W N$ be the set of down-blocks and neutral-blocks (see Fig. 4).

(ii) If $\mu(u)>\mu(v)$, then let $U P$ be the set of all up-blocks and neutral-blocks, and $D W N$ be the set of down-blocks (see Fig. 5).

(iii) If $\mu(u)=\mu(v)$, then split each neutral-block $[p, q]$ into two parts by the line segment $p q$, and put the upper-left part into $U P$ and the other in $D W N$. Then put all up-blocks in $U P$, and all down-blocks in $D W N$ (see Fig. 6).

The set of all points in the blocks in $U P$ forms a connected region, which we denoted by $R_{u p}$. Similarly, $R_{d w n}$ is defined by $D W N$.

Note that points in any (half) neutral-block, which is shown by a (half) white block in Figs. 4-6, belong to $R_{\text {up }}$ or $R_{\mathrm{dwn}}$. From the definition, we observe the next property.

Lemma 3. Let $R_{\mathrm{up}}$ and $R_{\mathrm{dwn}}$ be the regions defined for an ordered pair of nodes $u$ and $v$ in $V$. Then there is a monotone path $P$ that separates $S$ into $R_{\mathrm{up}}$ and $R_{\mathrm{dwn}}$, and it holds

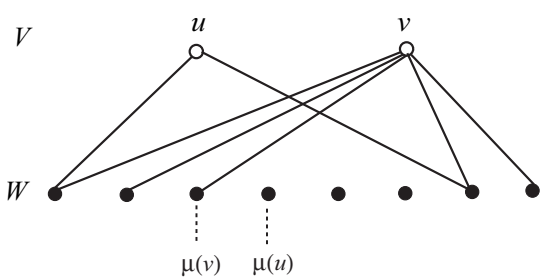

(a)

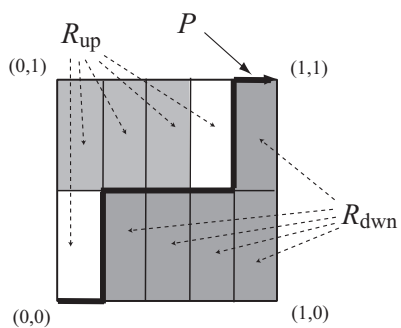

(b)

Fig. 5. (a) Two nodes $u$ and $v$ in the top layer. (b) A $(u, v)$-path $P$ of a $(2,5)$-sliced square $S$ in the case of (ii). 


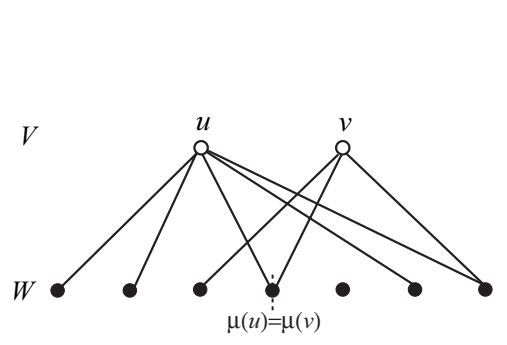

(a)

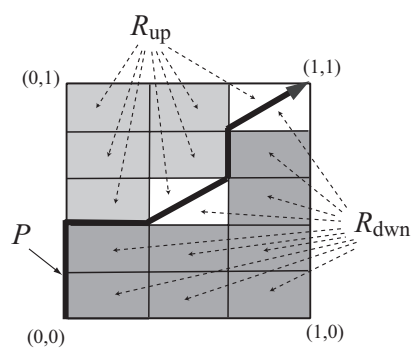

(b)

Fig. 6. (a) Two nodes $u$ and $v$ in the top layer. (b) A $(u, v)$-path $P$ of a (5,3)-sliced square $S$ in the case of (iii).

that

$$
a\left(R_{\mathrm{up}}\right)= \begin{cases}\frac{c_{u v}}{d_{u} d_{v}} & \text { if } \mu(u)<\mu(v), \\ \frac{c_{u v}+\Delta_{u v} / 2}{d_{u} d_{v}} & \text { if } \mu(u)=\mu(v), \\ \frac{c_{u v}+\Delta_{u v}}{d_{u} d_{v}} & \text { if } \mu(u)>\mu(v) .\end{cases}
$$

Moreover, $R_{\mathrm{up}}$ or $P$ contains point $(0.5,0.5)$ if $\mu(u) \geq \mu(v)$.

Such a path $P$ in the lemma is called the $(u, v)$-path with respect to $G$ and $\sigma$.

Lemma 4. Let $u, v \in V$ be two nodes in $(G, \sigma)$ such that $1 \leq c_{u v}<c_{v u}$ and $c_{u v}<$ $2 \Delta_{u v}$. Then $\mu(u)<\mu(v)$ holds unless $u$ and $v$ satisfy one of conditions (1)-(3):

$$
\begin{gathered}
c_{u v}=3, c_{v u}=4, d_{u}=d_{v}=3 \text { and } \Delta_{u v}=2, \\
c_{u v}=3, c_{v u}=5,\left\{d_{u}, d_{v}\right\}=\{2,5\} \text { and } \Delta_{u v}=2(\text { see Fig. 5), } \\
c_{u v}=5, c_{v u}=7,\left\{d_{u}, d_{v}\right\}=\{3,5\} \text { and } \Delta_{u v}=3 .
\end{gathered}
$$

Proof. See the Appendix.

We close this section by showing some technical lemmas.

Lemma 5. For constants $a>0, b, c>0$ and $d$ such that $a d-b c \geq 0$, function $f(x)=(a x+b)(1 /(c x+d)-2)$ takes the maximum $(1 / c)(\sqrt{a}-\sqrt{2(a d-b c)})^{2}$ over $x$ with $c x+d>0$.

Proof.

$$
(a x+b)\left(\frac{1}{c x+d}-2\right)=\frac{a x+b}{c x+d}-2 a x-2 b
$$




$$
\begin{aligned}
= & -\left(\sqrt{\frac{a d / c-b}{c x+d}}-\sqrt{\frac{2 a}{c}(c x+d)}\right)^{2} \\
& +\frac{1}{c}(\sqrt{a}-\sqrt{2(a d-b c)})^{2} \\
\leq & \frac{1}{c}(\sqrt{a}-\sqrt{2(a d-b c)})^{2} .
\end{aligned}
$$

Lemma 6. For four positive constants $a, b, c$ and $d$ with $b / a<d \leq 1 / \sqrt{2 c}$, function $f(x)=(a x-b)^{2}\left(1 / c x^{2}-2\right)(b / a<x \leq d)$ takes the maximum at $x=$ $\min \left\{d,(b / 2 a c)^{1 / 3}\right\}$.

Proof. $\quad d f / d x=2 a(a x-b)\left(1 / c x^{2}-2\right)+(a x-b)^{2} \cdot-2 / c x^{3}=2(a x-b)\left(-2 a+b / c x^{3}\right)$. By $a x-b>0$, the $f$ takes the maximum when $x=\min \left\{d,(b / 2 a c)^{1 / 3}\right\}$.

\section{Algorithm and Analysis}

In this paper we propose a randomized algorithm for the one-sided crossing minimization. We describe an outline of the algorithm here. For each node $u \in V$, the algorithm constructs an ordering $\pi$ of nodes in $V$ as a solution by aligning nodes $u \in V$ with respect to their neighbours $\kappa(u) \in \Gamma(u)$, where a neighbour $\kappa(u)$ of each node $u$ is determined by the following procedure. We first choose some interval $I_{u}=(s, t] \subseteq(0,1]$ for each node $u \in V$ according to a probabilistic scheme, where a value $z \in(0,1]$ corresponds the $\left\lceil z d_{u}\right\rceil$ th neighbour of $u$. We then choose a value $\theta(u)$ randomly from $I_{u}$, and set $\kappa(u)$ to be the $\left\lceil\theta(u) d_{u}\right\rceil$ th neighbour of $u$. In the following, we describe how to choose intervals $I_{u}$.

Let $\theta: V \rightarrow(0,1]$ be a function from $V$ to the set of reals in $(0,1]$, where $\theta(u)$ is called the real key of node $u$. Given a real-key function $\theta$, we construct a permutation $\pi_{\theta}$ of $\{1,2, \ldots,|V|\}$ by the next procedure.

\section{$\operatorname{PERMUTE}\left(\theta ; \pi_{\theta}\right)$}

Step 1. For each node $u \in V$, compute $j=\left\lceil\theta(u) d_{u}\right\rceil$ and define an integer key $\kappa(u)$ of $u$ by

$$
\kappa(u):=w_{j} \quad \text { for the } j \text { th neighbour } \quad w_{j} \in \Gamma(u),
$$

where $\Gamma(u)=\left\{w_{1}, w_{2}, \ldots, w_{d_{u}}\right\}\left(w_{1}<w_{2}<\cdots<w_{d_{u}}\right)$.

Step 2. Sort nodes $u \in V$ in the lexicographical order with respect to $(\kappa(u), \mu(u))$, where the ties among nodes $u$ with the same key $(\kappa(u), \mu(u))$ are broken as follows. For each set $\left\{u_{1}^{\prime}, u_{2}^{\prime}, \ldots, u_{\ell}^{\prime}\right\}$ of nodes that have the same key $(\kappa(u), \mu(u))$, choose as an ordering of them one of $u_{1}^{\prime}, u_{2}^{\prime}, \ldots, u_{\ell}^{\prime}$ and $u_{\ell}^{\prime}, u_{\ell+1}^{\prime}, \ldots, u_{1}^{\prime}$ randomly (where the indexing $i$ of $u_{i}^{\prime}$ is arbitrary). We denote by $\pi_{\theta}$ the resulting permutation of $\{1,2, \ldots,|V|\}$.

We see the following important property. 
Lemma 7. For two nodes $u, v \in V$, let $R_{\mathrm{up}}$ and $R_{\mathrm{dwn}}$ be the regions in Definition 2. Then for a given real-key function $\theta, \pi_{\theta}(u)<\pi_{\theta}(v)$ if point $(\theta(u), \theta(v))$ is inside $R_{\mathrm{dwn}}$ and $\pi_{\theta}(u)>\pi_{\theta}(v)$ if point $(\theta(u), \theta(v))$ is inside $R_{\mathrm{up}}$.

A scheme, based on which we choose a real-key function $\theta$ probabilistically, is defined by a set of tuples of reals $\mathcal{S}=\left\{\left(s_{i}, t_{i}, p_{i}\right) \mid i=1,2, \ldots, h\right\}$, such that $0<s_{i} \leq t_{i}<1$ and $0 \leq p_{i}$ for $i=1,2, \ldots, h$ and $\sum_{1 \leq i \leq h} p_{i}=1$, where we call each $\left(s_{i}, t_{i}, p_{i}\right)$ a subscheme. Given a scheme $\mathcal{S}$, we choose a real-key function $\theta$ in the following manner.

\section{RANDOM-KEY $(\mathcal{S} ; \theta)$}

Step 1 . Choose a subscheme $\left(s_{i}, t_{i}, p_{i}\right) \in \mathcal{S}$ with probability $p_{i}$.

Step 2. For each node $u \in V$, choose a real key $\theta(u)$ from $\left(s_{i}, t_{i}\right]$ uniformly.

We denote by $E_{\mathcal{S}}\left[\operatorname{cross}\left(u, v ; \pi_{\theta}\right)\right]$ and $E_{\mathcal{S}}\left[\operatorname{cross}\left(\pi_{\theta}\right)\right]$ respectively the expectations of $\operatorname{cross}\left(u, v ; \pi_{\theta}\right)$ and $\operatorname{cross}\left(\pi_{\theta}\right)$ over all real-key functions $\theta$ resulting from RANDOMKEY. In this paper we prove the next result.

Theorem 8. There is a scheme $\mathcal{S}$ such that $E_{\mathcal{S}}\left[\operatorname{cross}\left(\pi_{\theta}\right)\right] \leq 1.4664 L B$.

By the linearity of expectations, if we have a constant $\alpha \geq 1$ such that

$$
E_{\mathcal{S}}\left[\operatorname{cross}\left(u, v ; \pi_{\theta}\right)\right] \leq \alpha \min \left\{c_{u v}, c_{v u}\right\}, \quad u, v \in V,
$$

then it holds that $E_{\mathcal{S}}\left[\operatorname{cross}\left(\pi_{\theta}\right)\right] \leq \alpha L B$.

In the rest of this paper we fix two nodes $u, v \in V$, and analyse $E_{\mathcal{S}}\left[\operatorname{cross}\left(u, v ; \pi_{\theta}\right)\right]$ for a given scheme $\mathcal{S}$. Since the case of $\max \left\{c_{u v}, c_{v u}\right\} \leq 1.46 \min \left\{c_{u v}, c_{v u}\right\}$ needs no special consideration to prove Theorem 8, we consider nodes $u$ and $v$ such that $1.46 c_{u v}<c_{v u}$ or $1.46 c_{v u}<c_{u v}$. Without loss of generality we may assume that $d_{u} \leq d_{v}$ by renaming $u$ and $v$ and that $1.46 c_{u v}<c_{v u}$ by reversing the permutation $\sigma$ if necessary. Note that neither (1) nor (3) holds since 1.46 $c_{u v}<c_{v u}$. Moreover, we can assume that $c_{u v} \geq 1$ since otherwise (i.e., $\left.c_{u v}=0\right) \pi_{\theta}(u)<\pi_{\theta}(v)$ holds in any permutation $\pi_{\theta}$ computed by PERMUTE due to the first comparison of $\kappa(u)$ and $\kappa(v)$ (and the second comparison of $\mu(u)$ and $\mu(v)$ even if $\kappa(u)=\kappa(v))$.

For a given scheme $\mathcal{S}$ and a region $R \subseteq S$, let $p_{\mathcal{S}}(R)$ denote the probability that point $(\theta(u), \theta(v))$ falls inside $R$. By Lemma 7, we observe the next formula.

Lemma 9. $E_{\mathcal{S}}\left[\operatorname{cross}\left(u, v ; \pi_{\theta}\right)\right]=p_{\mathcal{S}}\left(R_{\mathrm{dwn}}\right) c_{u v}+p_{\mathcal{S}}\left(R_{\mathrm{up}}\right) c_{v u}$.

We are ready to derive an important inequality. 
Lemma 10. Assume that $1 \leq c_{u v}<c_{v u} / 1.46$ holds. Then it holds that

$$
\frac{E_{\mathcal{S}}\left[\operatorname{cross}\left(u, v ; \pi_{\theta}\right)\right]}{\min \left\{c_{u v}, c_{v u}\right\}} \leq\left\{\begin{array}{l}
1+p_{\mathcal{S}}\left(R_{\mathrm{up}}\right)\left(\frac{1}{a\left(R_{u p}\right)}-2\right) \\
1+p_{\mathcal{S}}\left(R_{\mathrm{up}}\right)\left(\frac{1.5}{a\left(R_{u p}\right)}-2.5\right)
\end{array} \quad \text { if } \quad \mu(u)<\mu(v), \quad \mu(u) \geq \mu(v),\right.
$$

unless (2) holds.

Proof. By Lemma 9, we get

$$
\begin{aligned}
\frac{E_{\mathcal{S}}\left[\operatorname{cross}\left(u, v ; \pi_{\theta}\right)\right]}{\min \left\{c_{u v}, c_{v u}\right\}} & =\frac{p_{\mathcal{S}}\left(R_{\mathrm{dwn}}\right) c_{u v}+p_{\mathcal{S}}\left(R_{\mathrm{up}}\right) c_{v u}}{c_{u v}} \\
& =\frac{\left(1-p_{\mathcal{S}}\left(R_{\mathrm{up}}\right)\right) c_{u v}+p_{\mathcal{S}}\left(R_{\mathrm{up}}\right)\left(d_{u} d_{v}-c_{u v}-\Delta_{u v}\right)}{c_{u v}} \\
& =1+p_{\mathcal{S}}\left(R_{\mathrm{up}}\right)\left(\frac{d_{u} d_{v}-\Delta_{u v}}{c_{u v}}-2\right) .
\end{aligned}
$$

First consider the case of $\mu(u)<\mu(v)$. By Lemma 3, we have $a\left(R_{\text {up }}\right)=c_{u v} / d_{u} d_{v}$. Hence

$$
\frac{1}{c_{u v}}\left(d_{u} d_{v}-\Delta_{u v}\right)-2=\frac{1}{c_{u v}}\left(\frac{c_{u v}}{a\left(R_{\mathrm{up}}\right)}-\Delta_{u v}\right)-2 \leq \frac{1}{a\left(R_{\mathrm{up}}\right)}-2 .
$$

Next consider the case of $\mu(u) \geq \mu(v)$. By Lemma 3, we have $a\left(R_{\text {up }}\right) \leq\left(c_{u v}+\right.$ $\left.\Delta_{u v}\right) / d_{u} d_{v}$. Since $1 \leq c_{u v}<c_{v u}$ holds but (2) does not hold for the $u$ and $v$, Lemma 4 implies that $\Delta_{u v} \leq c_{u v} / 2$. Then

$$
\begin{aligned}
\frac{1}{c_{u v}}\left(d_{u} d_{v}-\Delta_{u v}\right)-2 & \leq \frac{1}{c_{u v}}\left(\frac{c_{u v}+\Delta_{u v}}{a\left(R_{\mathrm{up}}\right)}-\Delta_{u v}\right)-2=\frac{1}{c_{u v}}\left(\frac{c_{u v}+\Delta_{u v}\left(1-a\left(R_{\mathrm{up}}\right)\right)}{a\left(R_{u p}\right)}\right)-2 \\
& \leq \frac{1}{c_{u v}}\left(\frac{c_{u v}+\frac{1}{2} c_{u v}\left(1-a\left(R_{\mathrm{up}}\right)\right)}{a\left(R_{\mathrm{up}}\right)}\right)-2=\frac{1.5}{a\left(R_{\mathrm{up}}\right)}-2.5 .
\end{aligned}
$$

This completes the proof.

We wish to find an optimal scheme $\mathcal{S}$ that minimizes $\max _{u, v \in V} E_{\mathcal{S}}\left[\operatorname{cross}\left(u, v ; \pi_{\theta}\right)\right] /$ $\min \left\{c_{u v}, c_{v u}\right\}$ (even though finding such an $\mathcal{S}$ analytically seems hard).

For this, we consider the set of all monotone paths $P$ for a given scheme $\mathcal{S}$. Let $P$ be an arbitrary monotone path between points $(0,0)$ and $(1,1)$ in a unit square $S$ (not necessarily a $(u, v)$-path for particular nodes $u, v \in V)$. Define $R_{\text {up }}(P)$ and $R_{\mathrm{dwn}}(P)$ to be the regions obtained by splitting $S$ with $P$, where we assume that $R_{\text {up }}(P)$ is above $R_{\mathrm{dwn}}(P)$. Let

$$
\beta(\mathcal{S}, P):= \begin{cases}p_{\mathcal{S}}\left(R_{\text {up }}(P)\right)\left(\frac{1}{a\left(R_{u p}(P)\right)}-2\right) & \text { if } \quad(0.5,0.5) \notin R_{\text {up }}(P), \\ \left.p_{\mathcal{S}}\left(R_{\text {up }}(P)\right)\left(\frac{1.5}{a\left(R_{u p}(P)\right.}\right)-2.5\right) & \text { if } \quad(0.5,0.5) \in R_{\text {up }}(P),\end{cases}
$$


and $\beta(\mathcal{S}):=\max \{\beta(\mathcal{S}, P) \mid$ monotone path $P\}$. Given a scheme $\mathcal{S}$, a monotone path $P$ from $(0,0)$ to $(1,1)$ in a unit square $S$ is called $\mathcal{S}$-maximal if $\beta(\mathcal{S}, P)=\beta(\mathcal{S})$.

Since the choice of monotone paths $P$ is relaxed, we obtain $E_{\mathcal{S}}\left[\operatorname{cross}\left(\pi_{\theta}\right)\right] \leq(1+$ $\beta(\mathcal{S})) L B$. (Recall that $(0.5,0.5) \in R_{\text {up }}$ holds if $\mu(u) \geq \mu(v)$ by Lemma 3.) Let $\beta^{*}=$ $\min \{\beta(\mathcal{S}) \mid$ schemes $\mathcal{S}\}$. Therefore, to prove Theorem 8, it suffices to show that $\beta^{*}<$ 0.4664 , i.e., there exists a scheme $\mathcal{S}$ such that $\beta(\mathcal{S})<0.4664$ (provided the case of (2) is treated separately to prove Theorem 8).

\section{A Scheme $\mathcal{S}$}

In this section we present a scheme $\mathcal{S}$ that achieves Theorem 8. We consider scheme

$$
\mathcal{S}=\left\{\left(s_{1}=0.0957, t_{1}=0.5, p_{1}=0.5\right),\left(s_{2}=0.5, t_{2}=0.9043, p_{2}=0.5\right)\right\} .
$$

Denote the squares $S_{1}=\left[\left(s_{1}, s_{1}\right),(0.5,0.5)\right]$ and $S_{2}=\left[(0.5,0.5),\left(t_{2}, t_{2}\right)\right]$, and the corners of these squares by $A_{1}=(0.0957,0.0957), A_{2}=(0.5,0.5), A_{3}=(0.9043,0.9043)$, $B_{1}=(0.5,0.0957), B_{2}=(0.9043,0.5), C_{1}=(0.0957,0.5)$ and $C_{2}=(0.5,0.9043)$. (The constant 0.0957 and others have been determined through some computational experiment.) Figure 7 illustrates this scheme. For simplicity, a square $S_{i}$ corresponding to a subscheme $\left(s_{i}, t_{i}, p_{i}\right)$ is called a subscheme.

We first consider the case of (2).

Lemma 11. For nodes $u, v \in V$ satisfying (2), it holds that $E_{\mathcal{S}}\left[\operatorname{cross}\left(u, v ; \pi_{\theta}\right)\right]<$ $1.4664 c_{u v}$.

Proof. For such $u$ and $v, c_{u v}=3, c_{v u}=5$ and $p_{\mathcal{S}}\left(R_{\mathrm{up}}\right)=p_{\mathcal{S}}\left(R_{\mathrm{dwn}}\right)=0.5$ hold. By Lemma $9, E_{\mathcal{S}}\left[\operatorname{cross}\left(u, v ; \pi_{\theta}\right)\right]=p_{\mathcal{S}}\left(R_{\mathrm{dwn}}\right) c_{u v}+p_{\mathcal{S}}\left(R_{\mathrm{up}}\right) c_{v u}=4<1.4664 c_{u v}$.

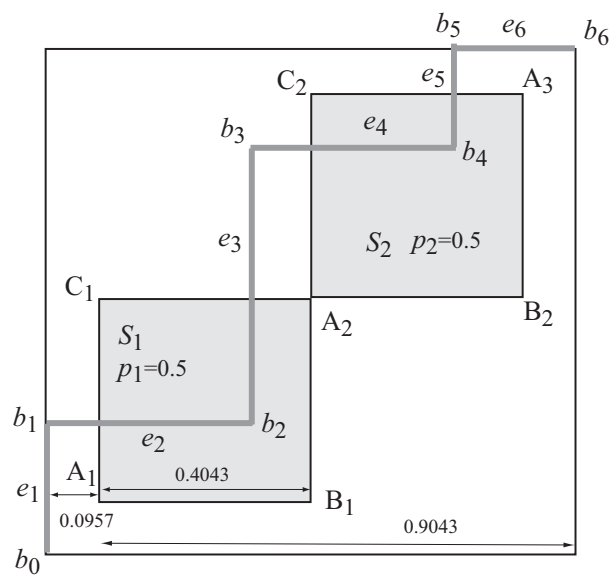

Fig. 7. Illustration of scheme $\mathcal{S}=\{(0.0957,0.5,0.5),(0.5,0.9043,0.5)\}$, where a grey line indicates an example of a monotone path $P$. 


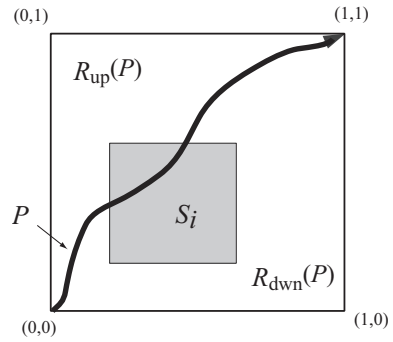

(a)

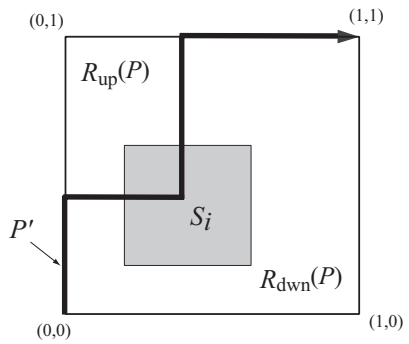

(b)

Fig. 8. A monotone path $P$ that passes through a square $S_{i}$.

Now consider nodes $u$ and $v$ in the general case. It is not difficult to see that an $\mathcal{S}$ maximal monotone path $P$ consists of axis-parallel line segments, and that the resulting region $R_{\text {up }}(P)$ contains at most one convex corner in each subscheme $S_{i}(i=1,2)$. For simplicity, we consider a single subscheme $S_{i}$. It should be noted that $p_{\mathcal{S}}\left(R_{\text {up }}\right)$ (or the contribution from $S_{i}$ to $\left.p_{\mathcal{S}}\left(R_{\text {up }}\right)\right)$ is given by $a\left(S_{i} \cap R_{\text {up }}(P)\right) / a\left(S_{i}\right)$. As shown in Fig. 8(a), if a monotone path $P$ does not satisfy these properties, then we can modify the path $P$ into another monotone path $P^{\prime}$ such that $a\left(S_{i} \cap R_{\text {up }}\left(P^{\prime}\right)\right)=a\left(S_{i} \cap R_{\text {up }}(P)\right)$ and $a\left(R_{\text {up }}\left(P^{\prime}\right)\right) \leq a\left(R_{\text {up }}(P)\right)$. For such an axis-parallel piecewise linear monotone path $P$, we denote the sequence of the corner points by

$$
b_{0}=(0,0), b_{1}, \ldots, b_{k}=(1,1),
$$

and the sequence of the edges by

$$
e_{1}=b_{0} b_{1}, e_{2}=b_{1} b_{2}, \ldots, e_{k}=b_{k-1} b_{k}
$$

(see Fig. 7). Let $e$ be an edge on a path $P$, where $e$ may be a partial segment of some edge $e_{i}$. Without loss of generality we further assume that an $\mathcal{S}$-maximal monotone path $P$ is chosen so that the number of edges of squares in subschemes or of the entire unit square that are overlapped by the edges in $P$ is maximized among all $\mathcal{S}$-maximal monotone paths.

We define the gain of edge $e$ at a subscheme $S_{i}=\left(s_{i}, t_{i}, p_{i}\right) \in \mathcal{S}$ as follows. Consider how much the amount of $p_{\mathcal{S}}\left(R_{\mathrm{up}}\right)$ changes if we move the line segment $e$ in its orthogonal direction by an infinitesimally small amount $\varepsilon$. The change in $p_{\mathcal{S}}\left(R_{\text {up }}\right)$ is

$$
\frac{\varepsilon \cdot \ell\left(e \cap S_{i}\right) \cdot p_{i}}{\left(t_{i}-s_{i}\right)^{2}}
$$

where $\ell\left(e \cap S_{i}\right)$ means the length of the intersection of $e$ and $S_{i}$. On the other hand, the change in $a\left(R_{\text {up }}(P)\right)$ is

$$
\varepsilon \cdot \ell(e)
$$

The gain is defined by the ratio of these two, i.e.,

$$
g(e)=\frac{\ell\left(e \cap S_{i}\right) \cdot p_{i}}{\left(t_{i}-s_{i}\right)^{2} \cdot \ell(e)} .
$$




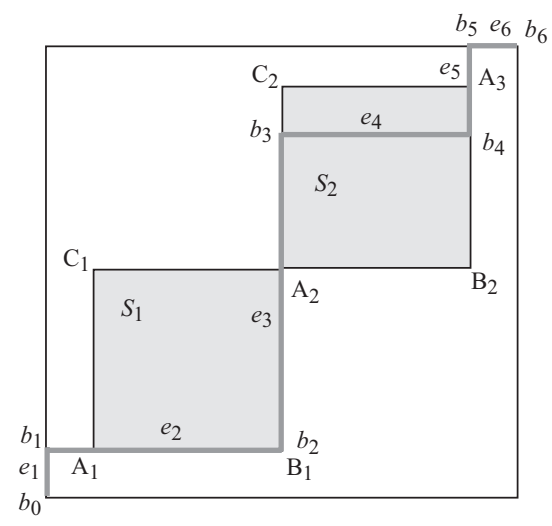

Fig. 9. Illustration of a piecewise linear monotone path $P$.

A vertical line segment $e$ on a path $P$ is called incrementable (resp., decrementable) if:

- There is a real $\delta>0$ such that $e$ has the same gain $g(e)$ (with respect to a subscheme $S_{i}$ ) after translating it rightward (resp., leftward) by any amount $\delta^{\prime} \in[0, \delta]$ (i.e., $e$ remains intersecting $S_{i}$ ).

- For the rectangle $R$ formed between $e$ and the translated edge $e^{\prime}$ and the current path $P$, there is a monotone path $P^{\prime}$ such that $R_{\text {up }}\left(P^{\prime}\right)=R_{\text {up }}(P) \cup R$ (resp., $\left.R_{\text {up }}\left(P^{\prime}\right)=R_{\text {up }}(P)-R\right)$.

Analogously, the incrementability (resp., decrementability) of a horizontal line segment $e$ is defined by replacing "rightward" with " downward" (resp., "leftward" with "upward"). In Fig. 9, for example, edges $e_{3}, A_{2} b_{3}$ and $e_{4}$ are incrementable, and $e_{5}, e_{2}$ and $A_{1} B_{1}$ are decrementable.

An edge $e_{i}$ between two corners in a path $P$ is called a free edge if it does not overlap with any edge of square $S_{i}$ in a subscheme or of the entire unit square $S$. For example, the free edges in Fig. 7 are $e_{2}, e_{3}, e_{4}$ and $e_{5}$, and the free edge in Fig. 9 is $e_{4}$.

By definition, we observe the following.

Lemma 12. For an $\mathcal{S}$-maximal monotone path $P$, let e and $e^{\prime}$ be respectively an incrementable edge and a decrementable edge such that $(0.5,0.5)$ is not an internal point in any of $e$ and $e^{\prime}$. If $e$ and $e^{\prime}$ are not adjacent, then $g(e)<g\left(e^{\prime}\right)$. If $e$ and $e^{\prime}$ are adjacent, then $g(e)=g\left(e^{\prime}\right)$.

Proof. Otherwise we would have another monotone path $P^{\prime}$ such that $\beta\left(\mathcal{S}, P^{\prime}\right)>$ $\beta(\mathcal{S}, P)$ or such that $\beta\left(\mathcal{S}, P^{\prime}\right)=\beta(\mathcal{S}, P)$ and $P^{\prime}$ overlaps with more edges of the squares than $P$ does.

In particular, there is no pair of non-adjacent free edges in an $\mathcal{S}$-maximal monotone path $P$. 
In what follows $P$ is assumed to be an $\mathcal{S}$-maximal monotone path, and for simplicity $R_{\text {up }}(P)$ is written by $R_{\text {up }}$. To prove that $\beta(\mathcal{S}, P) \leq 0.4664$ holds for our scheme $\mathcal{S}$, we distinguish the following cases:

Case A. Point $(0.5,0.5)$ is not on the boundary of $R_{\text {up }}$ or inside $R_{\text {up }} ; \beta(\mathcal{S}, P)$ is given by $p_{\mathcal{S}}\left(R_{\text {up }}\right)\left(1 / a\left(R_{\text {up }}\right)-2\right)$.

Case B. Point $(0.5,0.5)$ is on the boundary of $R_{\text {up }}$ or inside $R_{\text {up }} ; \beta(\mathcal{S}, P)$ is given by $p_{\mathcal{S}}\left(R_{u p}\right)\left(1.5 / a\left(R_{u p}\right)-2.5\right)$.

Case (1) $R_{\text {up }}$ contains an internal point from exactly one of $S_{1}$ and $S_{2}$; without loss of generality $R_{\text {up }}$ contains no internal point in $S_{2}$.

Case (2) $R_{\text {up }}$ contains an internal point from each of $S_{1}$ and $S_{2}$.

\subsection{Case A(1)}

In this case, $R_{\text {up }}$ has no convex corner in $S_{2}$, and exactly one convex corner $b_{3}$ in $S_{1}$ (see Fig. 10(a)). Consider edges $e_{2}=b_{1} b_{2}$ and $e_{3}=b_{2} b_{3}$ in $P$. By $(0.5,0.5) \in R_{\text {up }}, e_{3}$ does not overlap with $B_{1} A_{2}$, and thereby $e_{3}$ is a free edge. Let $x=\ell\left(e_{2}\right)$ and $\bar{y}=\ell\left(e_{3}\right)$.

First consider the case where $e_{2}$ does not overlap with $A_{1} B_{1}$, i.e., $e_{2}$ is a free edge. Then $g\left(e_{2}\right)=0.5 /(0.4043)^{2} \times(x-0.0957) / x$, and $g\left(e_{3}\right)=0.5 /(0.4043)^{2} \times(\bar{y}-0.5) / \bar{y}$. Since $P$ is $\mathcal{S}$-maximal, it must hold that $g\left(e_{2}\right)=g\left(e_{3}\right)$ for two free edges. Thus we have $\bar{y}=(0.5 / 0.0957) x$. Hence by $\bar{y} \leq 0.9043$, we have $x<0.9043 \times 0.0957 / 0.5=$ 0.17308302 . By $\bar{y}=(0.5 / 0.0957) x$, we have $\bar{y}-0.5=(0.5 / 0.0957) x-0.5=$ $(0.5 / 0.0957)(x-0.0957)$. We have $a\left(R_{\mathrm{up}}\right)=x \bar{y}$ and

$$
\begin{aligned}
p_{\mathcal{S}}\left(R_{\mathrm{up}}\right) & =0.5 \times \frac{(x-0.0957)(\bar{y}-0.5)}{(0.4043)^{2}} \\
& =\frac{0.5 \times 0.5}{(0.4043)^{2} \times 0.0957}(x-0.0957)^{2}
\end{aligned}
$$

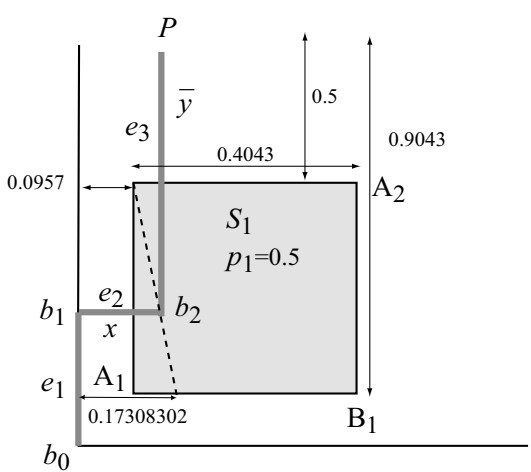

(a)

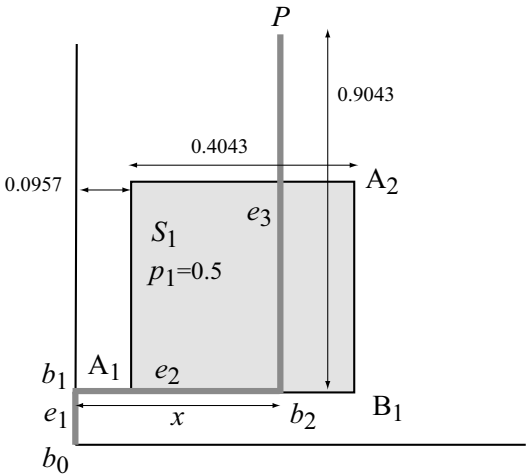

(b)

Fig. 10. Illustration for Case $\mathrm{A}(1)$, where (a) indicates the case where a corner point of $P$ is inside $S_{1}$, and (b) indicates the case where edge $A_{1} B_{1}$ of $S_{1}$ is overlapped by edge $e_{2}$ of $P$. 
Then

$$
\begin{aligned}
\beta(\mathcal{S}, P) & =p_{\mathcal{S}}\left(R_{\mathrm{up}}\right)\left(\frac{1}{a\left(R_{\text {up }}\right)}-2\right) \\
& =\frac{0.5 \times 0.5}{(0.4043)^{2} \times 0.0957}(x-0.0957)^{2}\left(\frac{1}{(0.5 / 0.0957) x^{2}}-2\right) .
\end{aligned}
$$

By Lemma 6 with $a=1, b=0.0957$ and $c=0.5 / 0.0957$, this takes the maximum at $x=\min \left\{0.17308302,(0.0957 /(2 \times 0.5 / 0.0957))^{1 / 3}\right\}$ (the latter is at least 0.209$)$.

Since the maximum is attained at $x=0.17308302$, we only have to consider the case when $b_{2}$ is on edge $A_{1} B_{1}$ (see Fig. 10(b)). Then $a\left(R_{\text {up }}\right)=0.9043 x$ and $p_{\mathcal{S}}\left(R_{\text {up }}\right)=$ $0.5 \times(x-0.0957) / 0.4043$ hold. For $x \in(0.0957,0.5)$,

$$
\beta(\mathcal{S}, P)=p_{\mathcal{S}}\left(R_{\text {up }}\right)\left(\frac{1}{a\left(R_{\text {up }}\right)}-2\right)=\frac{0.5}{0.4043}(x-0.0957)\left(\frac{1}{0.9043 x}-2\right) .
$$

By Lemma 5 with $a=1, b=-0.0957, c=0.9043$ and $d=0$, we have

$$
\begin{aligned}
p_{\mathcal{S}}\left(R_{\mathrm{up}}\right)\left(\frac{1}{a\left(R_{\mathrm{up}}\right)}-2\right) & \leq \frac{0.5}{0.4043} \times \frac{1}{c}(\sqrt{a}-\sqrt{2(a d-b c)})^{2} \\
& =\frac{0.5}{0.4043} \times \frac{1}{0.9043}(\sqrt{1}-\sqrt{2 \times 0.0957 \times 0.9043})^{2} \\
& <0.46638
\end{aligned}
$$

\subsection{Case $\mathrm{A}(2)$}

In this case, $A_{2}=(0.5,0.5)$ is not in $R_{\text {up }}$, but $R_{\text {up }}$ has a convex corner in each $S_{i}$. Then we see that $e_{3}$ and $e_{4}$ are free edges. Since $e_{3}$ and $e_{4}$ are free edges, there is no other free edge by Lemma 12 . Hence edges $e_{2}$ and $e_{5}$ must overlap with $A_{1} B_{1}$ and $B_{2} A_{3}$, respectively (see Fig. 11).

By Lemma 12, edges $e_{3}$ and $e_{4}$ have the same gain, i.e., $\ell\left(e_{3}\right)=\ell\left(e_{4}\right)$. Let $t=$ $\ell\left(e_{4}\right) \in(0.4043,0.8086)$. Then we have $a\left(R_{\text {up }}\right)=(0.9043)^{2}-t^{2}$, and $p_{\mathcal{S}}\left(R_{\text {up }}\right)=$ $2 \times 0.5 / 0.4043 \times(0.8086-t)(\leq 2 \times 0.5)$. Then we have $\beta(\mathcal{S}, P)=p_{\mathcal{S}}\left(R_{\text {up }}\right)\left(1 / a\left(R_{u p}\right)-\right.$ $2)<0.4664$ for $t \in(0.4043,0.8086)$. (For example, to prove this, repeat the following computation after initializing a variable $p$ for $p_{\mathcal{S}}\left(R_{\text {up }}\right)$ as $p:=1$ :

$R:=\frac{1}{0.4663 / p+2} ; \quad t:=\sqrt{(0.9043)^{2}-R} ; \quad p:=2 \times \frac{0.5}{0.4043} \times(0.8086-t)$.

After a finite number of iterations, $x$ becomes greater than 0.8086 , which implies that there is no $t \in(0.4043,0.8086)$ such that $\beta(\mathcal{S}, P) \geq 0.4664$.)

\subsection{Case $\mathrm{B}(1)$}

In this case, $A_{2}=(0.5,0.5)$ is on the path $P$ by the $\mathcal{S}$-maximality. 


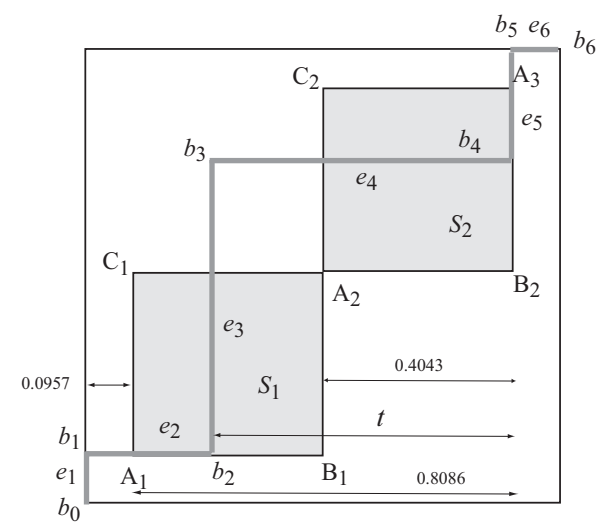

Fig. 11. Illustration for an $\mathcal{S}$-maximal path $P$ in Case $\mathrm{A}(2)$.

First assume that $e_{3}$ does not overlap with $B_{1} A_{2}$. In this case, $b_{3}$ is on $C_{1} A_{2}$, i.e., $e_{4}=$ $b_{3} A_{2}$. However, edge $e_{4}=b_{3} A_{2}$ is incrementable, $e_{2}$ is decrementable and $g\left(b_{3} A_{2}\right)>$ $g\left(e_{2}\right)$ holds. By Lemma 12 this contradicts the $\mathcal{S}$-maximality of path $P$.

Then assume that $e_{3}$ overlaps with $B_{1} A_{2}$ (see Fig. 12). By letting $t=\ell\left(e_{3}\right)$, we have $a\left(R_{\text {up }}\right)=0.5 t+0.25$ and $p_{\mathcal{S}}\left(R_{\text {up }}\right)=0.5 \times t / 0.4043$. Then

$$
\begin{aligned}
\beta(\mathcal{S}, P) & =p_{\mathcal{S}}\left(R_{\text {up }}\right)\left(\frac{1.5}{a\left(R_{\text {up }}\right)}-2.5\right)=0.5 \times \frac{t}{0.4043}\left(\frac{1.5}{0.5 t+0.25}-2.5\right) \\
& =0.5 \times \frac{t}{0.4043}\left(\frac{1}{0.5 t+0.25} \times \frac{6}{5}-2\right) \times \frac{5}{4} \\
& =1.545881771 \times t\left(\frac{1}{\frac{5}{12} t+\frac{5}{24}}-2\right),
\end{aligned}
$$

which is at most 0.46626 by Lemma 5 with $a=1.545881771, b=0, c=\frac{5}{12}$ and $d=\frac{5}{24}$.

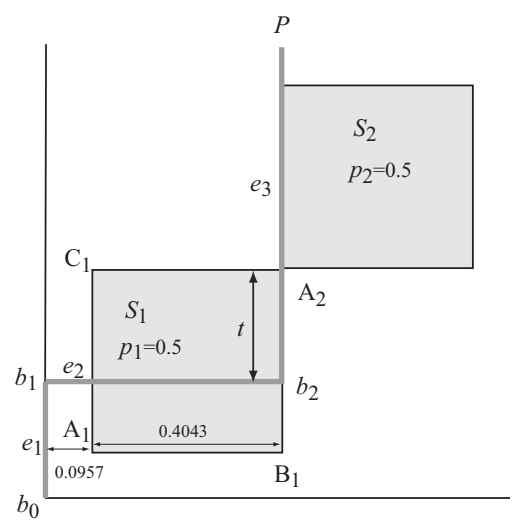

Fig. 12. Illustration for an $\mathcal{S}$-maximal path $P$ in Case $\mathrm{B}(1)$, where $e_{3}$ overlaps with $B_{1} A_{2}$. 


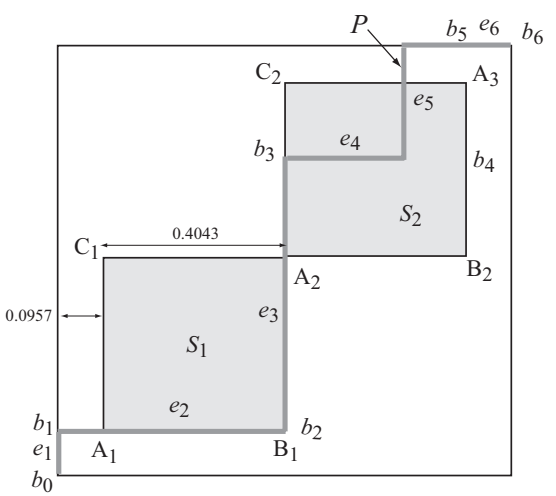

(a)

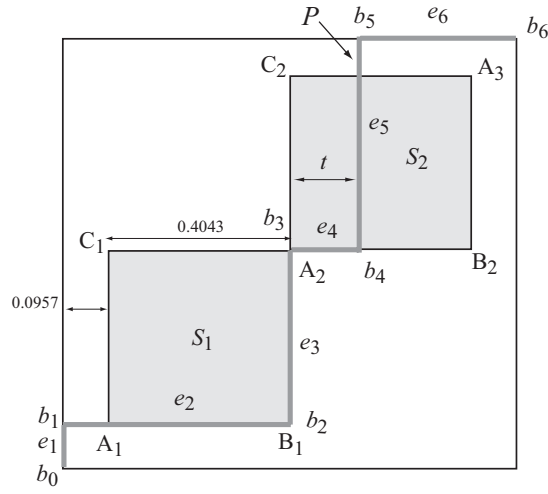

(b)

Fig. 13. Illustration for a possible $\mathcal{S}$-maximal path $P$ in Case $\mathrm{B}(2)$, where (a) indicates the case where $B_{1} A_{2}$ and $A_{2} C_{2}$ are overlapped by some edges in $P$, and (b) indicates the case where $B_{1} A_{2}$ and $A_{2} B_{2}$ are overlapped by some edges in $P$.

\subsection{Case $\mathrm{B}(2)$}

Since $R_{\text {up }}$ contains an internal point from each of $S_{1}$ and $S_{2}$, one of the following holds:

(i) $C_{1} A_{2}$ and $A_{2} C_{2}$ are overlapped by some edges in $P$.

(ii) $B_{1} A_{2}$ and $A_{2} C_{2}$ are overlapped by some edges in $P$ (the case where $C_{1} A_{2}$ and $A_{2} B_{2}$ are overlapped can be treated symmetrically). (See Fig. 13(a).)

(iii) $B_{1} A_{2}$ and $A_{2} B_{2}$ are overlapped by some edges in $P$. (See Fig. 13(b).)

We show that the first two cases cannot yield an $\mathcal{S}$-maximal path $P$. In (i) there must be at least two non-adjacent free edges in $P$. In (ii), $e_{4}$ and $e_{5}$ are incrementable and decrementable and $g\left(e_{4}\right)>g\left(e_{5}\right)$. Thus, by Lemma $12, P$ in (i) and (ii) is not $\mathcal{S}$-maximal.

Consider (iii), where $e_{2}$ overlaps with $A_{1} B_{1}$ or $e_{5}$ overlaps with $B_{2} A_{3}$ (otherwise both would be non-adjacent free edges); the former case is assumed without loss of generality (see Fig. 13(b)).

By letting $t=\ell\left(e_{4}\right)$, we have $a\left(R_{\text {up }}\right)=0.5 t+0.5 \times 0.9043=0.5 t+0.45215$ and $p_{\mathcal{S}}\left(R_{\text {up }}\right)=0.5 \times t / 0.4043+0.5$. Then

$$
\begin{aligned}
\beta(\mathcal{S}, P) & =p_{\mathcal{S}}\left(R_{\mathrm{up}}\right)\left(\frac{1.5}{a\left(R_{u p}\right)}-2.5\right)=\left(0.5 \times \frac{t}{0.4043}+0.5\right)\left(\frac{1.5}{0.5 t+0.45215}-2.5\right) \\
& =\left(0.5 \times \frac{t}{0.4043}+0.5\right)\left(\frac{1}{0.5 t+0.45215} \times \frac{6}{5}-2\right) \frac{5}{4} \\
& =(1.545881771 t+0.625)\left(\frac{1}{\frac{5}{12} t+0.36425625}-2\right),
\end{aligned}
$$

which is at most 0.46626 by Lemma 5 with $a=1.545881771, b=0.625, c=\frac{5}{12}$ and $d=0.45215 \times \frac{5}{6}$.

This establishes $\beta(\mathcal{S})<0.4664$ and hence Theorems 8 and 1 . 


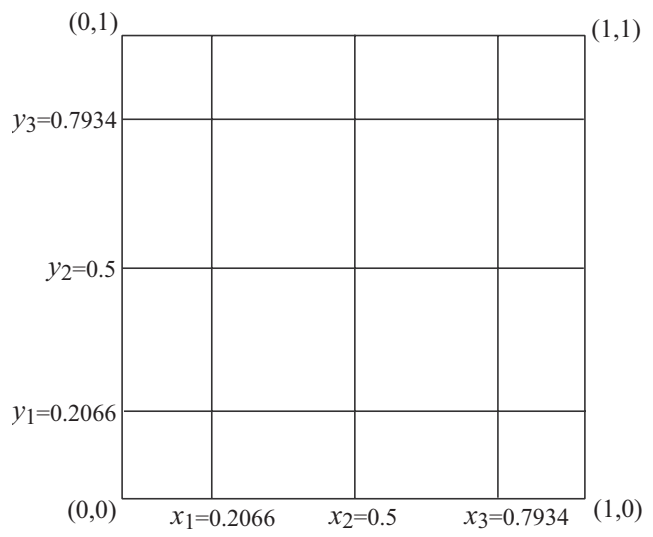

Fig. 14. A partition of a unit square $S$.

\section{Lower Bound on $\beta^{*}$}

One may consider whether there is a better scheme $\mathcal{S}^{\prime}$ that has $\beta\left(\mathcal{S}^{\prime}\right)$ much smaller than 0.4667 . In this section we show, however, that the scheme $\mathcal{S}$ in the previous section is nearly optimal by proving that there is no scheme $\mathcal{S}^{\prime}$ with $\beta\left(\mathcal{S}^{\prime}\right)<0.4414$. That is, we prove the next result.

Theorem 13. $0.4414<\beta^{*}<0.4667$.

Since we have shown $\beta^{*} \leq 0.4667$, we now estimate $\beta^{*}$ from below. Let $\mathcal{S}$ be an arbitrary scheme. For $x_{1}=y_{1}=0.2066, x_{2}=y_{2}=0.5$ and $x_{3}=y_{3}=1-x_{1}$, we partition a unit square $S$ into 16 blocks by three vertical lines with $x$-coordinates $x_{1}, x_{2}$ and $x_{3}$ and three horizontal lines with $y$-coordinates $y_{1}, y_{2}$ and $y_{3}$ (see Fig. 14). We consider the following eight monotone paths:

$$
\begin{aligned}
& P_{1}=\left\langle(0,0),\left(0, y_{2}\right),\left(x_{3}, y_{2}\right),\left(x_{3}, 1\right),(1,1)\right\rangle, \\
& P_{2}=\left\langle(0,0),\left(0, y_{1}\right),\left(x_{2}, y_{1}\right),\left(x_{2}, 1\right),(1,1)\right\rangle, \\
& P_{3}=\left\langle(0,0),\left(x_{1}, 0\right),\left(x_{1}, 1\right),(1,1)\right\rangle, \\
& P_{4}=\left\langle(0,0),\left(0, y_{3}\right),\left(1, y_{3}\right),(1,1)\right\rangle, \\
& P_{1}^{\prime}=\left\langle(0,0),\left(x_{1}, 0\right),\left(x_{1}, y_{2}\right),\left(1, y_{2}\right),(1,1)\right\rangle, \\
& P_{2}^{\prime}=\left\langle(0,0),\left(x_{2}, 0\right),\left(x_{2}, y_{3}\right),\left(1, y_{3}\right),(1,1)\right\rangle, \\
& P_{3}^{\prime}=\left\langle(0,0),\left(x_{3}, 0\right),\left(x_{3}, 1\right),(1,1)\right\rangle, \\
& P_{4}^{\prime}=\left\langle(0,0),\left(0, y_{1}\right),\left(1, y_{1}\right),(1,1)\right\rangle .
\end{aligned}
$$

Let $a_{1}=0.5 \times(1-0.2066)$ and $a_{2}=0.2066$. Then we have

$$
\begin{aligned}
& a\left(R_{\mathrm{up}}\left(P_{1}\right)\right)=a\left(R_{\mathrm{dwn}}\left(P_{1}^{\prime}\right)\right)=a\left(R_{\mathrm{up}}\left(P_{2}\right)\right)=a\left(R_{\mathrm{dwn}}\left(P_{2}^{\prime}\right)\right)=a_{1}, \\
& a\left(R_{\mathrm{up}}\left(P_{3}\right)\right)=a\left(R_{\mathrm{dwn}}\left(P_{3}^{\prime}\right)\right)=a\left(R_{\mathrm{up}}\left(P_{4}\right)\right)=a\left(R_{\mathrm{dwn}}\left(P_{4}^{\prime}\right)\right)=a_{2} .
\end{aligned}
$$


Observe that each block in $S$ is contained in at least two regions from $\left\{R_{\text {up }}\left(P_{1}\right), \ldots\right.$, $\left.R_{\text {up }}\left(P_{4}\right), R_{\mathrm{dwn}}\left(P_{1}^{\prime}\right), \ldots, R_{\mathrm{dwn}}\left(P_{4}^{\prime}\right)\right\}$. Therefore, it holds that

$$
\sum_{i=1}^{4} p_{\mathcal{S}}^{\text {up }}\left(P_{i}\right)+\sum_{i=1}^{4} p_{\mathcal{S}}^{\text {dwn }}\left(P_{i}^{\prime}\right) \geq 2
$$

By definition, $\beta^{*}$ satisfies

$$
\begin{aligned}
& p_{\mathcal{S}}^{\text {up }}\left(P_{i}\right)\left(\frac{1.5}{a\left(R_{\mathrm{up}}\left(P_{i}\right)\right)}-2.5\right) \leq \beta^{*} \quad(i=1,2), \\
& p_{\mathcal{S}}^{\text {up }}\left(P_{i}\right)\left(\frac{1}{a\left(R_{\mathrm{up}}\left(P_{i}\right)\right)}-2\right) \leq \beta^{*} \quad(i=3,4) .
\end{aligned}
$$

Similarly, by considering path $P_{i}^{\prime}$ as a monotone path from $(1,1)$ to $(0,0)$, we have

$$
\begin{gathered}
p_{\mathcal{S}}^{\mathrm{dwn}}\left(P_{i}^{\prime}\right)\left(\frac{1.5}{a\left(R_{\mathrm{dwn}}\left(P_{i}^{\prime}\right)\right)}-2.5\right) \leq \beta^{*} \quad(i=1,2), \\
p_{\mathcal{S}}^{\mathrm{dwn}}\left(P_{i}^{\prime}\right)\left(\frac{1}{a\left(R_{\mathrm{dwn}}\left(P_{i}^{\prime}\right)\right)}-2\right) \leq \beta^{*} \quad(i=3,4) .
\end{gathered}
$$

Hence it holds that

$$
\begin{aligned}
\sum_{i=1}^{4} p_{\mathcal{S}}^{\mathrm{up}}\left(P_{i}\right) & +\sum_{i=1}^{4} p_{\mathcal{S}}^{\mathrm{dwn}}\left(P_{i}^{\prime}\right) \\
\leq & \beta^{*} \sum_{i=1,2} \frac{1}{1.5 / a\left(R_{\mathrm{up}}\left(P_{i}\right)\right)-2.5}+\beta^{*} \sum_{i=3,4} \frac{1}{1 / a\left(R_{\mathrm{up}}\left(P_{i}\right)\right)-2} \\
& +\beta^{*} \sum_{i=1,2} \frac{1}{1.5 / a\left(R_{\mathrm{dwn}}\left(P_{i}^{\prime}\right)\right)-2.5}+\beta^{*} \sum_{i=3,4} \frac{1}{1 / a\left(R_{\mathrm{dwn}}\left(P_{i}^{\prime}\right)\right)-2} \\
= & \beta^{*}\left(\frac{4}{1.5 / a_{1}-2.5}+\frac{4}{1 / a_{2}-2}\right) .
\end{aligned}
$$

From this, we have

$$
\beta^{*} \geq \frac{\sum_{i=1}^{4} p_{\mathcal{S}}^{\text {up }}\left(P_{i}\right)+\sum_{i=1}^{4} p_{\mathcal{S}}^{\mathrm{dwn}}\left(P_{i}^{\prime}\right)}{4 /\left(1.5 / a_{1}-2.5\right)+4 /\left(1 / a_{2}-2\right)} \geq \frac{2}{4 /\left(1.5 / a_{1}-2.5\right)+4 /\left(1 / a_{2}-2\right)}>0.4414,
$$

as required.

\section{Deterministic Algorithms}

Based on the algorithm in Section 3, in this section we present deterministic algorithms. Let $n_{1}=|V|, n_{2}=|W|$ and $m=|E|$. We first review the following result. 
Lemma 14 [13]. The number of crossings in a two-layered drawing $(\pi, \sigma)$ can be computed in $O\left(\min \left\{n_{1} n_{2}, m \log \left(\min \left\{n_{1}, n_{2}\right\}\right)\right\}\right)$ time.

In what follows we assume that nodes in $W$ are numbered according to a given permutation $\sigma$. Here we observe how to compute $\{\mu(u) \mid u \in V\},\left\{c_{u v}, c_{v u} \mid u, v \in V\right\}$ and area $R_{\text {up }}$ for each pair $u, v \in V$. For each node $u \in V, \mu(u)$ can be computed in $O\left(d_{u}\right)$ time by using a linear time median algorithm. By computing a sorted ordering of $\Gamma(u) \cup \Gamma(v)$ in $O\left(\left(d_{u}+d_{v}\right) \log \left(d_{u}+d_{v}\right)\right)$ time, we can identify the $(u, v)$-path $P_{u v}$ with respect to $G$ and $\sigma$. Based on $P_{u v}$, we can compute $c_{u v}, c_{v u}$ and $R_{\text {up }}$ for $u, v \in V$ in $O\left(d_{u}+d_{v}\right)$ time. The total time for computing these values is $O\left(\sum\left(d_{u}+\right.\right.$ $\left.\left.d_{v}\right) \log \left(d_{u}+d_{v}\right)\right)=O\left(m n_{1} \log n_{1}\right)$. For a scheme $\mathcal{S}$ which consists of a single subscheme $(s, t, 1)$, we can compute $E_{\mathcal{S}}\left[\operatorname{cross}\left(u, v ; \pi_{\theta}\right)\right]=p_{\mathcal{S}}\left(R_{\mathrm{dwn}}\right) c_{u v}+p_{\mathcal{S}}\left(R_{\mathrm{up}}\right) c_{v u}$ for each pair $u, v \in V$ in $O\left(d_{u}+d_{v}\right)$ time, and the expected crossing number $E_{\mathcal{S}}\left[\operatorname{cross}\left(\pi_{\theta}\right)\right]=$ $\sum_{u, v \in V} E_{\mathcal{S}}\left[\operatorname{cross}\left(u, v ; \pi_{\theta}\right)\right]$ in $O\left(\sum\left(d_{u}+d_{v}\right)\right)=O\left(m n_{1}\right)$ time. Based on this, we prove the following.

Theorem 15. For a given scheme $\mathcal{S}=\left\{\left(s_{i}, t_{i}, p_{i}\right) \mid i=1,2, \ldots, h\right\}, \operatorname{PERMUTE}\left(\theta ; \pi_{\theta}\right)$ can be derandomized into an $O\left(\left(h+n_{1}\right) n_{1} m\right)$ time deterministic algorithm that delivers a permutation $\pi$ of $V$ with $\operatorname{cross}(\pi) \leq E_{\mathcal{S}}\left[\operatorname{cross}\left(\pi_{\theta}\right)\right]$.

Let $\mathcal{S}=\left\{S_{i}=\left(s_{i}, t_{i}, p_{i}\right) \mid i=1,2, \ldots, h\right\}$. We can derandomize PERMUTE $\left(\theta ; \pi_{\theta}\right)$ as follows. We first determine which subscheme $S_{i} \in \mathcal{S}$ will construct an ordering $\pi$ of $V$ whose expected crossing number is at most $E_{\mathcal{S}}\left[\operatorname{cross}\left(\pi_{\theta}\right)\right]$. Let $S_{k}$ be such a subscheme (i.e., $\left.E_{\left(s_{k}, t_{k}, 1\right)}\left[\operatorname{cross}\left(\pi_{\theta}\right)\right] \leq E_{\mathcal{S}}\left[\operatorname{cross}\left(\pi_{\theta}\right)\right]\right)$. Then we consider $\theta(u) \in\left(s_{k}, t_{k}\right]$ for a node $u \in V$. Since a real key $x=\theta(u)$ will be mapped to an integer key $\left\lceil x d_{u}\right\rceil \in\left[1, d_{u}\right]$, we can partition interval $\left(s_{k}, t_{k}\right]$ into subintervals such that any $\theta(u)$ chosen from a subinterval is mapped to the same integer. Then we can choose a subinterval for $\theta(u)$ which will construct an ordering whose crossing number is at most the current expected crossing number $E_{\left(s_{k}, t_{k}, 1\right)}\left[\operatorname{cross}\left(\pi_{\theta}\right)\right]$. By repeating this for all nodes in $V$, we choose the best subinterval for each node $u \in V$, from which the lexicographical order with respect to $(\kappa(u), \mu(u))$ is uniquely determined except for subsets of nodes $u$ with the same key $(\kappa(u), \mu(u))$. We can break a tie for each such subset by choosing a better one from two possible orderings for the subset (where one is the reverse of the other). All the above procedures are deterministic, and the resulting ordering $\pi$ of $V$ has a crossing number less than or equal to $E_{\mathcal{S}}\left[\operatorname{cross}\left(\pi_{\theta}\right)\right]$. The entire algorithm can be described as follows.

\section{DERANDOMIZE $(\mathcal{S}, G, \sigma)$}

Step 1 (Choose the best subscheme). For each subscheme $\left(s_{i}, t_{i}, p_{i}\right) \in \mathcal{S}$, consider scheme $\mathcal{S}_{i}=\left\{\left(s_{i}, t_{i}, 1\right)\right\}$, and compute $E_{\mathcal{S}_{i}}\left[\operatorname{cross}\left(u, v ; \pi_{\theta_{i}}\right)\right]=p_{\mathcal{S}_{i}}\left(R_{\mathrm{dwn}}\right) c_{u v}+$ $p_{\mathcal{S}_{i}}\left(R_{\mathrm{up}}\right) c_{v u}$ for every pair $u, v \in V$, and $E_{\mathcal{S}_{i}}\left[\operatorname{cross}\left(\pi_{\theta_{i}}\right)\right]=\sum_{u, v \in V} E_{\mathcal{S}_{i}}\left[\operatorname{cross}\left(u, v ; \pi_{\theta_{i}}\right)\right]$, where $\pi_{\theta_{i}}$ denotes an ordering of $V$ which will be obtained by scheme $\mathcal{S}_{i}$. Then choose a scheme $\mathcal{S}_{k}=\left\{\left(s_{k}, t_{k}, 1\right)\right\}$ that has the minimum expected number of crossings $E_{\mathcal{S}_{i}}\left[\operatorname{cross}\left(\pi_{\theta_{i}}\right)\right]$ among $\mathcal{S}_{i}, i=1,2, \ldots, h$. 
Step 2. (Choose the best subinterval for each $\theta(u)$ ). Let $V^{\prime}:=V ; s:=s_{k} ; t:=t_{k}$; $I_{v}:=(s, t]$ for all $v \in V ; \mathcal{I}:=\left\{I_{v} \mid v \in V\right\}$

while $V^{\prime} \neq \varnothing$ do

Choose a node $u \in V^{\prime}$, and let $V^{\prime}:=V^{\prime}-u$; We change $I_{u}$ with a subinterval of $I_{u}$ as follows. Partition $I_{u}=(s, t]$ into subintervals

$$
I_{1}^{\prime}=(s, \bar{s}], I_{2}^{\prime}=\left(\bar{s}, \bar{s}+1 / d_{u}\right], I_{3}^{\prime}=\left(\bar{s}+1 / d_{u}, \bar{s}+2 / d_{u}\right], \ldots, I_{q}^{\prime}=(\underline{t} t],
$$

so that all values $x$ in each subinterval $I_{j}^{\prime}$ are mapped to the same integer $\left\lceil x d_{u}\right\rceil \in$ $\left[1, d_{u}\right]$ (where $\bar{s}=\left(\left\lfloor s d_{u}\right\rfloor+1\right) / d_{u}, \underline{t}=\left(\left\lceil t d_{u}\right\rceil-1\right) / d_{u}$ and $\left.q=\left\lceil t d_{u}\right\rceil-\left\lfloor s d_{u}\right\rfloor\right)$. For each $I_{j}^{\prime}$, by setting $I_{u}:=I_{j}^{\prime}$ temporarily, we compute $E_{\mathcal{I}}\left[\operatorname{cross}\left(\pi_{\mathcal{I}}\right)\right]=$ $\sum_{u, v \in V} E_{\mathcal{I}}\left[\operatorname{cross}\left(u, v ; \pi_{\mathcal{I}}\right)\right]$, the expected number of crossings of an ordering $\pi_{\mathcal{I}}$ which will be constructed by PERMUTE with uniform random real keys $\theta(v) \in I_{v} \in \mathcal{I}, v \in V$. Then fix $I_{u}$ to be the subscheme $I_{j}^{\prime}$ that minimizes $E_{\mathcal{I}}\left[\operatorname{cross}\left(\pi_{\mathcal{I}}\right)\right]$ among all subintervals $I_{j}^{\prime}$ of $I_{u}=(s, t]$.

Step 3. (Choose better tie-break). Note that, after fixing interval $I_{v}$ for all $v \in V$, PERMUTE now computes unique integer keys $\kappa(v), v \in V$. Sort nodes $u \in V$ in the lexicographical order with respect to $(\kappa(u), \mu(u))$, where the ties among nodes $u$ with the same key $(\kappa(u), \mu(u))$ are broken as follows. For each set $\left\{u_{1}^{\prime}, u_{2}^{\prime}, \ldots, u_{\ell}^{\prime}\right\}$ of nodes that have the same key $(\kappa(u), \mu(u))$, choose an ordering from $u_{1}^{\prime}, u_{2}^{\prime}, \ldots, u_{\ell}^{\prime}$ and $u_{\ell}^{\prime}, u_{\ell+1}^{\prime}, \ldots, u_{1}^{\prime}$ so as to minimize the number of crossings.

We can compute $p_{\mathcal{S}_{i}}$ and $p_{\mathcal{S}_{i}}\left(R_{\text {up }}\right)$ in $O\left(d_{u} d_{v}\right)$ time for each pair $u, v \in V$. Then Step 1 can be executed in $O\left(h \sum d_{u} d_{v}\right)=O\left(h m n_{1}\right)$ time, including the time to choose the best subscheme. In Step 2 let $u$ be a node chosen from $V^{\prime}$. Since $\left\{I_{j}^{\prime} \mid j=1,2, \ldots, q\right\}$ is a partition of $I_{u}$, it is not difficult to see that the expected number of crossings $E_{\mathcal{I}}\left[\operatorname{cross}\left(\pi_{\mathcal{I}}\right)\right]$ for all $I_{j}^{\prime}$ can be computed in $O\left(m n_{1}\right)$ time. Then Step 2 can be implemented to run in $O\left(m n_{1}^{2}\right)$ time. Step 3 can be executed in $O\left(m \log n_{1}\right)$ time, since a lexicographical order with respect to $(\kappa(u), \mu(u))$ can be obtained in $O\left(n_{1} \log n_{1}\right)$ time and one of the two orderings of each set $\left\{u_{1}^{\prime}, u_{2}^{\prime}, \ldots, u_{\ell}^{\prime}\right\}$ of nodes that have the same key $(\kappa(u), \mu(u))$ can be chosen in $O\left(\left(d_{u_{1}^{\prime}}+d_{u_{2}^{\prime}}+\cdots+d_{u_{\ell}^{\prime}}\right) \log n_{1}\right)$ time. This proves Theorem 15.

Based on a different scheme, we show a faster deterministic algorithm that computes a permutation $\pi$ with $\operatorname{cross}(\pi) \leq 1.803107 L B$ in $O\left(\min \left\{n_{1} n_{2}, m \log \left(\min \left\{n_{1}, n_{2}\right\}\right)\right\}\right)$ time. We consider a scheme $\mathcal{S}$ which consists of $2 k$ subschemes $S_{i}=\left(s_{i}, t_{i}, p_{i}\right)$, $i=1,2, \ldots, 2 k$, such that $s_{i}=t_{i}$ for $i=1,2, \ldots, 2 k$ and $s_{j}=s_{2 k+1-j}$ and $p_{j}=p_{2 k+1-j}$ for $j=k+1, k+2, \ldots, 2 k$. Note that, once we choose a subscheme $S_{i}$ in Step 1 of RANDOM_KEY, $\theta(u)=s_{i}$ holds for all nodes $u \in V$ in Step 2, and the permutation $\pi_{\theta}$ in PERMUTE can be computed in $O\left(n_{1}+n_{2}\right)$ time using bucket sort. Therefore, for the above type of scheme $\mathcal{S}$, we can obtain a permutation $\pi$ such that $\operatorname{cross}(\pi) \leq E_{\mathcal{S}}\left[\operatorname{cross}\left(\pi_{\theta}\right)\right]$ by choosing the best permutation among those $\pi_{\theta}$ computed for all subschemes $S_{i}$. Hence we can compute a permutation $\pi$ with $\operatorname{cross}(\pi) \leq E_{\mathcal{S}}\left[\operatorname{cross}\left(\pi_{\theta}\right)\right] \leq(1+\beta(\mathcal{S})) L B$ in $O\left(k \min \left\{n_{1} n_{2}, m \log \left(\min \left\{n_{1}, n_{2}\right\}\right)\right\}\right)$ time (provided that the case of condition (2) is treated separately). Also note that for any $\mathcal{S}$-maximal path $P$, the region $R_{\text {up }}(P)$ has convex corners from $\left\{\left(s_{i}, s_{i}\right) \mid 1 \leq i \leq 2 k\right\}$. Hence we only need to check $2^{2 k}$ such paths to estimate $\beta(\mathcal{S})$. 
For an example of such a scheme $\mathcal{S}$ with $k=12$, consider $s_{i}$ and $p_{i}(i=1,2, \ldots, 12)$ as follows: $s_{1}=0.02, p_{1}=\frac{0.1}{60.2}, s_{2}=0.06, p_{2}=\frac{1}{60.2}, s_{3}=0.10, p_{3}=\frac{2}{60.2}, s_{4}=0.14$, $p_{4}=\frac{3}{60.2}, s_{5}=0.18, p_{5}=\frac{3}{60.2}, s_{6}=0.22, p_{6}=\frac{3}{60.2}, s_{7}=0.26, p_{7}=\frac{3}{60.2}, s_{8}=0.30$, $p_{8}=\frac{3}{60.2}, s_{9}=0.34, p_{9}=\frac{3}{60.2}, s_{10}=0.38, p_{10}=\frac{3}{60.2}, s_{11}=0.42, p_{11}=\frac{3}{60.2}$, $s_{12}=0.46$ and $p_{12}=\frac{3}{60.2}$.

By inspecting all possible $2^{24} \mathcal{S}$-maximal paths, we can prove that $\beta(\mathcal{S})<0.803107$. In condition (2), $\max \left\{c_{u v}, c_{v u}\right\} / \min \left\{c_{u v}, c_{v u}\right\}=5 / 3<1.8$ holds. Therefore, for this scheme $\mathcal{S}$, the above deterministic algorithm delivers a 1.803107-approximation solution in $O\left(\min \left\{n_{1} n_{2}, m \log \left(\min \left\{n_{1}, n_{2}\right\}\right)\right\}\right)$ time.

\section{Concluding Remarks}

In this paper we have shown that the conventional lower bound $L B$ is much closer to the optimal one than the previous best estimation asserted. The proof is based on a probabilistic argument, which provides a randomized algorithm that delivers an approximation solution with the claimed performance guarantee 1.4664 . There may be some other scheme that achieves a better ratio. We, however, show that, for our current approach based on Lemma 10, no scheme achieves a performance guarantee better than 1.4414. Needless to say, this does not imply that the gap between the optimum and the lower bound is actually 1.4414. The current known gap is $13 / 11 \simeq 1.1818$, which is attained by the example in Fig. 2. The current estimation on $E_{\mathcal{S}}\left[\operatorname{cross}\left(u, v ; \pi_{\theta}\right)\right] / \min \left\{c_{u v}, c_{v u}\right\}$ in Lemma 10 depends on a pair of nodes $u$ and $v$ with rather small degrees. For a class of graphs with special structures such as regular graphs and graphs with a large minimum degree, it seems possible to get a better estimation on $E_{\mathcal{S}}\left[\operatorname{cross}\left(u, v ; \pi_{\theta}\right)\right] / \min \left\{c_{u v}, c_{v u}\right\}$, which would lead to an upper bound better than 1.4664. Recently, Nagamochi [12] proved that there always exits a solution whose crossing number is at most $(1.2964+12 /(\delta-4)) L B$ if the minimum degree $\delta$ of nodes in $V$ is at least 5 .

\section{Acknowledgement}

We express our gratitude to the anonymous referees for their valuable comments.

\section{Appendix. Proof of Lemma 4}

We assume that $d_{u} \leq d_{v}$ holds; by the definitions of $c_{u v}$ and $\mu$, the other case can be treated by reversing permutations $\pi$ and $\sigma$ and by exchanging node names $u$ and $v$. Let $u_{1}^{\prime}<u_{2}^{\prime}<\cdots<u_{d_{u}}^{\prime}$ and $v_{1}^{\prime}<v_{2}^{\prime}<\cdots<v_{d_{v}}^{\prime}$ be the nodes in $\Gamma(u)$ and $\Gamma(v)$, respectively, and let $w_{1}<w_{2}<\cdots<w_{\Delta_{u v}}$ be the nodes in $\Gamma(u) \cap \Gamma(v)$. Recall that $\Delta_{u v}\left(\Delta_{u v}-1\right) / 2 \leq \min \left\{c_{u v}, c_{v u}\right\}$ holds. By the assumption $c_{v u}>c_{u v}<2 \Delta_{u v}$, we have

$$
1 \leq \Delta_{u v} \leq 4
$$

since otherwise (if $\left.\Delta_{u v} \geq 5\right) 2 \Delta_{u v} \leq \Delta_{u v}\left(\Delta_{u v}-1\right) / 2$ would imply $2 \Delta_{u v} \leq \min \left\{c_{u v}, c_{v u}\right\}$. Since $2 \Delta_{u v} \leq \Delta_{u v}\left(\Delta_{u v}-1\right) / 2+3$ holds, we see that $2 \Delta_{u v} \leq c_{u v}$ holds if $\Delta_{u v}\left(\Delta_{u v}-\right.$ 


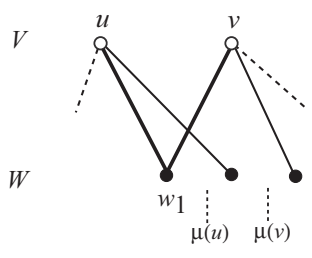

(a)

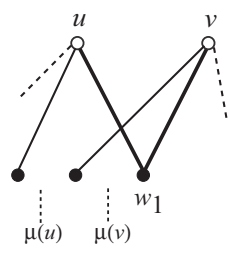

(b)

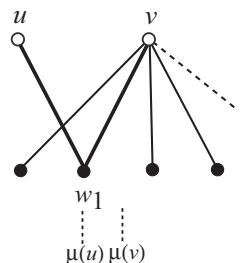

(c)

Fig. 15. Possible configurations for the case $\Delta_{u v}=1,1=c_{u v}<c_{v u}$ and $d_{u} \leq d_{v}$.

1) $/ 2+3 \leq c_{u v}$. Let $c_{u v} \leq \Delta_{u v}\left(\Delta_{u v}-1\right) / 2+2$. If $c_{u v}=\Delta_{u v}\left(\Delta_{u v}-1\right) / 2$, then we have $d_{u}>\Delta_{u v}$ or $d_{v}>\Delta_{u v}$ (since otherwise $d_{u}=d_{v}=\Delta_{u v}$ would imply $c_{u v}=c_{v u}$ ), indicating that $\mu(u)<\mu(v)$ holds. Let

$$
c_{u v}-\frac{\Delta_{u v}\left(\Delta_{u v}-1\right)}{2} \in\{1,2\}
$$

Case 1: $\Delta_{u v}=1$. Then $c_{u v}=1$ by the assumption that $1 \leq c_{u v}<2 \Delta_{u v}$. Consider the following three cases: (a) $w_{1}<u_{d_{u}}^{\prime}$, (b) $w_{1}=u_{d_{u}}^{\prime}$ and $d_{u} \geq 2$ and (c) $w_{1}=u_{d_{u}}^{\prime}$ and $d_{u}=1$ (see Fig. 15). In (a), by $c_{u v}=\Delta_{u v}=1, w_{1}=u_{d_{u}-1}^{\prime}=v_{1}^{\prime}$ holds, and by $d_{v} \geq d_{u} \geq 2, u_{d_{u}}^{\prime}<v_{2}^{\prime}$ holds, implying $\mu(u) \leq\left(w_{1}+u_{d_{u}}^{\prime}\right) / 2<\left(w_{1}+v_{2}^{\prime}\right) / 2 \leq \mu(v)$. In (b), by $c_{u v}=\Delta_{u v}=1, w_{1}=u_{d_{u}}^{\prime}=v_{2}^{\prime}$ holds, and by $d_{u} \geq 2, u_{d_{u}-1}^{\prime}<v_{1}^{\prime}$ holds, implying $\mu(u) \leq\left(u_{d_{u}-1}^{\prime}+w_{1}\right) / 2<\left(v_{1}^{\prime}+w_{1}\right) / 2 \leq \mu(v)$. In (c), by $c_{u v}=\Delta_{u v}=1, w_{1}=u_{1}^{\prime}=v_{2}^{\prime}$ holds, and by $c_{u v}<c_{v u}, d_{v} \geq 4$ holds, implying $\mu(u)=w_{1}<\left(w_{1}+v_{3}^{\prime}\right) / 2 \leq \mu(v)$.

Case 2: $\quad \Delta_{u v}=2$. First consider the case $c_{u v}-\Delta_{u v}\left(\Delta_{u v}-1\right) / 2=1$. Thus $c_{u v}=2$, where one crossing in $c_{u v}$ is created by $\operatorname{arcs}\left(u, w_{2}\right)$ and $\left(v, w_{1}\right)$ and the other is created by one of these arcs and an arc between $u$ (or $v$ ) and its neighbour $w^{\prime}$ with $w_{1}<w^{\prime}<w_{2}$. We distinguish four cases: (a) $w^{\prime}=u_{d_{u}-1}^{\prime}$ and $d_{u} \geq 4$, (b) $w^{\prime}=u_{d_{u}-1}^{\prime}$ and $d_{u}=3$, (c) $w^{\prime}=v_{2}^{\prime}$ and $d_{u} \geq 3$ and (d) $w^{\prime}=v_{2}^{\prime}$ and $d_{u}=2$ (see Fig. 16). In (d), $d_{v} \geq 4$ by $c_{u v}<c_{v u}$. We have $\mu(u) \leq\left(w_{1}+w^{\prime}\right) / 2<\left(w_{1}+w_{2}\right) / 2 \leq \mu(v)$ in (a), $\mu(u)=w^{\prime}<w_{2} \leq \mu(v)$ in (b), $\mu(u) \leq w_{1}<w^{\prime} \leq \mu(v)$ in (c) and $\mu(u)=\left(w_{1}+w_{2}\right) / 2<\left(w^{\prime}+w_{2}\right) / 2 \leq \mu(v)$ in $(\mathrm{d})$.

Next consider the case $c_{u v}-\Delta_{u v}\left(\Delta_{u v}-1\right) / 2=2$. Thus $c_{u v}=3$. One crossing in $c_{u v}=3$ is created by arcs $\left(u, w_{2}\right)$ and $\left(v, w_{1}\right)$. There are two cases: (e) the other two crossings are on an arc between $u$ (or $v$ ) and its neighbour $w^{\prime}$ with $w_{1}<w_{2}<w^{\prime}$ or

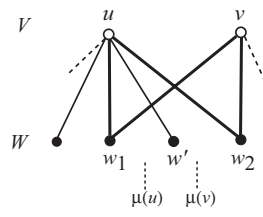

(a)

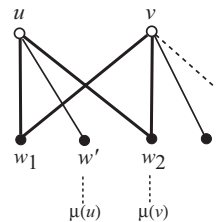

(b)

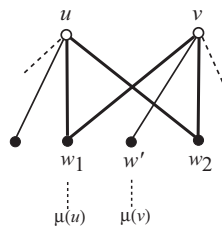

(c)

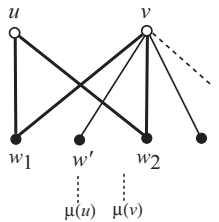

(d)

Fig. 16. Possible configurations for the case $\Delta_{u v}=2, c_{u v}-\Delta_{u v}\left(\Delta_{u v}-1\right) / 2=1$ and $d_{u} \leq d_{v}$. 


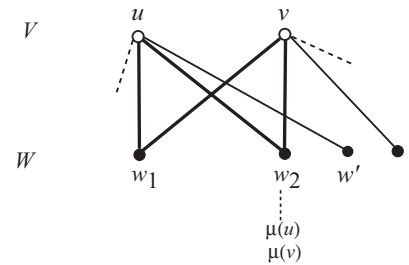

(e) $w_{2}<w^{\prime}$

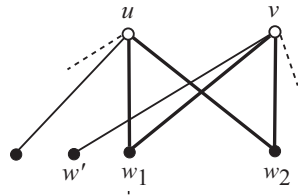

$\mu(u)$

$\mu(v)$

(e) $w^{\prime}<w_{2}$

Fig. 17. Possible configurations for case (e): $\Delta_{u v}=2, c_{u v}-\Delta_{u v}\left(\Delta_{u v}-1\right) / 2=2$ and $d_{u} \leq d_{v}$.

$w^{\prime}<w_{1}<w_{2}$ (see Fig. 17), and (f) the other two crossings are respectively on two arcs between $u$ (or $v$ ) and its neighbours $w^{\prime}$ and $w^{\prime \prime}$ with $w_{1}<w^{\prime}<w^{\prime \prime}<w_{2}$ (see Fig. 18). Consider case (e), where $d_{u} \geq 3$ by $c_{u v}<c_{v u}$. Then $c_{v u}=4$ and $\mu(u)=\mu(v)$ hold if $d_{u}=d_{v}=3$ (i.e., condition (1)); $\mu(u)<\mu(v)$ holds otherwise (i.e., $d_{v} \geq 4$ ).

Consider case (f). If $\left(u, w^{\prime}\right),\left(u, w^{\prime \prime}\right) \in E$, then $d_{v} \geq d_{u} \geq 4$ and $\mu(u) \leq\left(w^{\prime}+\right.$ $\left.w^{\prime \prime}\right) / 2<\left(w_{2}+v_{3}^{\prime}\right) \leq \mu(v)$ hold. If $\left(u, w^{\prime}\right),\left(v, w^{\prime \prime}\right) \in E$, then $\mu(u) \leq w^{\prime}<w^{\prime \prime} \leq \mu(v)$ holds. Let $\left(v, w^{\prime}\right),\left(v, w^{\prime \prime}\right) \in E$. If $d_{u} \geq 3$, then $\mu(u) \leq w_{1}<\left(w^{\prime}+w^{\prime \prime}\right) / 2 \leq \mu(v)$ holds. If $d_{u}=2$, then $d_{v} \geq 5$ (otherwise $d_{v}=4$ would imply $c_{v u}=2 \cdot 4-c_{u v}-\Delta_{u v}=3$, contradicting the assumption $\left.c_{u v}<c_{v u}\right)$. For $d_{u}=2$ and $d_{v}=5, \mu(u)=\left(w_{1}+\right.$ $\left.w_{2}\right) / 2 \geq w^{\prime \prime}=\mu(v)$ may hold (i.e., condition (2)). For $d_{u}=2$ and $d_{v} \geq 6$, we have $\mu(u)=\left(w_{1}+w_{2}\right) / 2<\left(w^{\prime \prime}+w_{2}\right) / 2 \leq \mu(v)$.

Case 3: $\quad \Delta_{u v}=3$. First consider the case $c_{u v}-\Delta_{u v}\left(\Delta_{u v}-1\right) / 2=1$. Thus $c_{u v}=4$, where three crossings in $c_{u v}$ are created by arcs $\left(u, w_{2}\right),\left(u, w_{3}\right),\left(v, w_{1}\right)$ and $\left(v, w_{2}\right)$, and the other is created by one of these arcs and an arc between $u$ (or $v$ ) and its neighbour $w^{\prime}$ with $w_{1}<w^{\prime}<w_{3}$. We distinguish two cases: (a) $w^{\prime}<w_{2}$ and (b) $w_{2}<w^{\prime}$ (see Fig. 19). We have $\mu(u) \leq\left(w^{\prime}+w_{2}\right) / 2<w_{2} \leq \mu(v)$ in (a), and $\mu(u) \leq w_{2}<$ $\left(w_{2}+w^{\prime}\right) / 2 \leq \mu(v)$ in (b).

Next consider the case $c_{u v}-\Delta_{u v}\left(\Delta_{u v}-1\right) / 2=2$. Thus $c_{u v}=5$. Three crossings in $c_{u v}$ are created by $\operatorname{arcs}\left(u, w_{2}\right),\left(u, w_{3}\right),\left(v, w_{1}\right)$ and $\left(v, w_{2}\right)$. There are two cases: (c) the other two crossings are on an arc between $u$ (resp., $v$ ) and its neighbour $w^{\prime}$ with $w_{2}<w^{\prime}<w_{3}$ (resp., $w_{1}<w^{\prime}<w_{2}$ ) (see Fig. 20), and (d) the other two crossings are respectively on two arcs between $u$ (or $v$ ) and its neighbours $w^{\prime}$ and $w^{\prime \prime}$ with $w_{1}<w^{\prime}<w_{2}<w^{\prime \prime}<w_{3}, w_{1}<w^{\prime}<w^{\prime \prime}<w_{2}$ or $w_{2}<w^{\prime}<w^{\prime \prime}<w_{3}$ (see Fig. 21). In (c) if $\left(u, w^{\prime}\right) \in E$, then by $d_{v} \geq d_{u} \geq 4, \mu(u) \leq\left(w_{2}+w^{\prime}\right) / 2<\left(w_{2}+w_{3}\right) / 2 \leq \mu(v)$. Consider the case $\left(v, w^{\prime}\right) \in E$ in (c). If $d_{u} \geq 4$, then $\mu(u) \leq\left(w_{1}+w_{2}\right) / 2<\left(w^{\prime}+\right.$ $\left.w_{2}\right) / 2 \leq \mu(v)$. If $d_{u}=3$ and $d_{v} \geq 6$, then $\mu(u)=w_{2}<\left(w_{2}+w_{3}\right) / 2 \leq \mu(v)$. For $d_{u}=3$ and $d_{v}=5$, we have $c_{v u}=7$ and $\mu(u)=\mu(v)=w_{2}$ (i.e., condition (3)).

Consider case (d). If $\left(u, w^{\prime}\right),\left(v, w^{\prime \prime}\right) \in E$, then $\mu(u) \leq\left(w^{\prime}+w_{2}\right) / 2<\left(w_{2}+w^{\prime \prime}\right) / 2 \leq$ $\mu(v)$ holds. If $\left(u, w^{\prime}\right),\left(u, w^{\prime \prime}\right) \in E$, then $d_{v} \geq d_{u} \geq 5$ and $\mu(u) \leq w^{\prime \prime}<w_{3} \leq \mu(v)$ hold. If $\left(v, w^{\prime}\right),\left(v, w^{\prime \prime}\right) \in E$, then $\mu(u) \leq w_{2}<w^{\prime} \leq \mu(v)$ holds.

Case 4: $\quad \Delta_{u v}=4$. By $c_{u v}-\Delta_{u v}\left(\Delta_{u v}-1\right) / 2 \in\{1,2\}, c_{u v} \in\{7,8\}$. There are the following seven cases: (a) $c_{u v}=7$, and $\left(u, w^{\prime}\right) \in E$ for a node $w^{\prime} \in W$ with $w_{1}<w^{\prime}<$ $w_{2}$, (b) $c_{u v}=7$, and $\left(v, w^{\prime}\right) \in E$ for a node $w^{\prime} \in W$ with $w_{3}<w^{\prime}<w_{4}$, (c) $c_{u v}=8$, and $\left(u, w^{\prime}\right) \in E$ for a node $w^{\prime} \in W$ with $w_{2}<w^{\prime}<w_{3}$, (d) $c_{u v}=8$, and $\left(v, w^{\prime}\right) \in E$ 


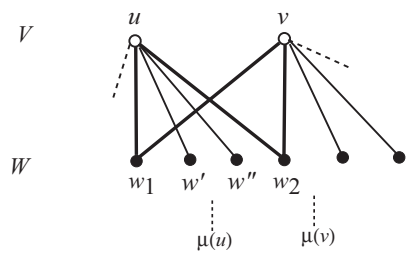

(f) $\left(u, w^{\prime}\right),\left(u, w^{\prime \prime}\right)$

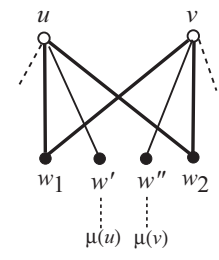

(f) $\left(u, w^{\prime}\right),\left(v, w^{\prime \prime}\right)$

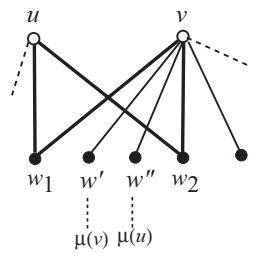

(f) $\left(v, w^{\prime}\right),\left(v, w^{\prime \prime}\right)$

Fig. 18. Possible configurations for case (f): $\Delta_{u v}=2, c_{u v}-\Delta_{u v}\left(\Delta_{u v}-1\right) / 2=2$ and $d_{u} \leq d_{v}$.

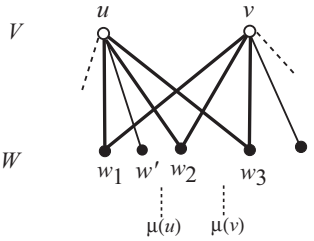

(a)

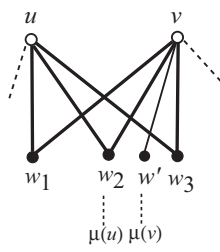

(b)

Fig. 19. Possible configurations for case $\Delta_{u v}=3, c_{u v}-\Delta_{u v}\left(\Delta_{u v}-1\right) / 2=1$ and $d_{u} \leq d_{v}$.

V

$W$

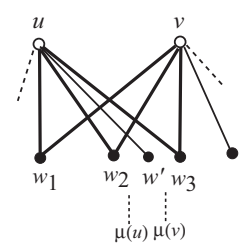

(c) $\left(u, w^{\prime}\right)$

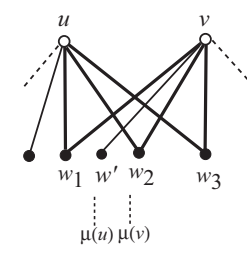

(c) $\left(v, w^{\prime}\right), d_{u}>3$

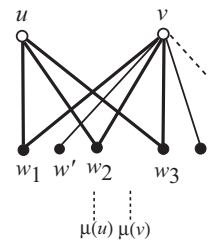

(c) $\left(v, w^{\prime}\right), d_{u}=3$

Fig. 20. Possible configurations for case (c): $\Delta_{u v}=3, c_{u v}-\Delta_{u v}\left(\Delta_{u v}-1\right) / 2=2$ and $d_{u} \leq d_{v}$.

V

$W$

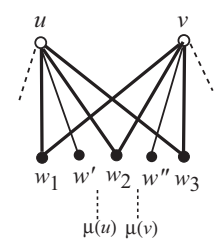

(d) $\left(u, w^{\prime}\right),\left(v, w^{\prime \prime}\right)$

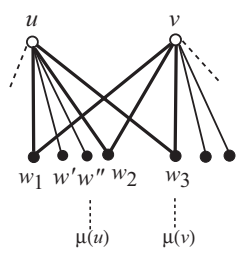

(d) $\left(u, w^{\prime}\right),\left(u, w^{\prime \prime}\right)$

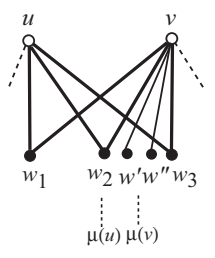

(d) $\left(v, w^{\prime}\right),\left(v, w^{\prime \prime}\right)$

Fig. 21. Possible configurations for the case (d): $\Delta_{u v}=3, c_{u v}-\Delta_{u v}\left(\Delta_{u v}-1\right) / 2=2$ and $d_{u} \leq d_{v}$. 


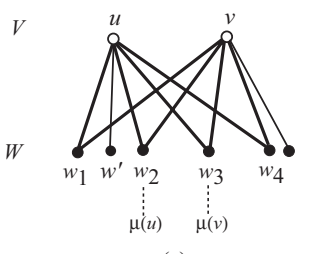

(a)

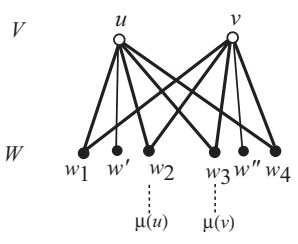

(e)

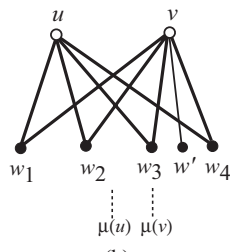

(b)

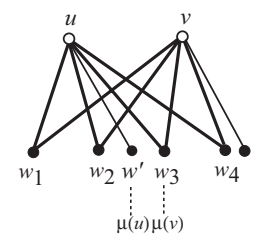

(f)

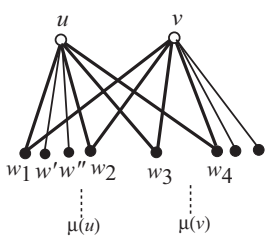

(c)

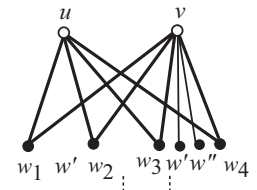

$\mu(u) \quad \mu(v)$

(d)

Fig. 22. Possible configurations for the case $\Delta_{u v}=4, c_{u v}-\Delta_{u v}\left(\Delta_{u v}-1\right) / 2 \in\{1,2\}$ and $d_{u} \leq d_{v}$.

for a node $w^{\prime} \in W$ with $w_{2}<w^{\prime}<w_{3}$, (e) $c_{u v}=8$, and $\left(u, w^{\prime}\right),\left(v, w^{\prime \prime}\right) \in E$ for nodes $w^{\prime}, w^{\prime \prime} \in W$ with $w_{1}<w^{\prime}<w_{2}<w_{3}<w^{\prime \prime}<w_{4}$, (f) $c_{u v}=8$, and $\left(u, w^{\prime}\right),\left(u, w^{\prime \prime}\right) \in E$ for a node $w^{\prime} w^{\prime \prime} \in W$ with $w_{1}<w^{\prime}<w^{\prime \prime}<w_{2}$, and (g) $c_{u v}=8$, and $\left(v, w^{\prime}\right),\left(v, w^{\prime \prime}\right) \in E$ for a node $w^{\prime} w^{\prime \prime} \in W$ with $w_{3}<w^{\prime}<w^{\prime \prime}<w_{4}$ (see Fig. 22).

We see that $\mu(u) \leq w_{2}<w_{3} \leq \mu(v)$ in (a), $\mu(u) \leq\left(w_{2}+w_{3}\right) / 2<w_{3} \leq \mu(v)$ in (b), $\mu(u) \leq\left(w^{\prime \prime}+w_{2}\right) / 2<\left(w_{3}+w_{4}\right) / 2 \leq \mu(v)$ in (c), $\mu(u) \leq\left(w_{2}+w_{3}\right) / 2<\left(w_{3}+\right.$ $\left.w^{\prime}\right) / 2 \leq \mu(v)$ in (d), $\mu(u) \leq w_{2}<w_{3} \leq \mu(v)$ in (e), and $\mu(u) \leq w^{\prime}<w_{3} \leq \mu(v)$ in (f), respectively. In (g), $d_{u} \geq 5$ or $d_{v} \geq 6$ since otherwise $c_{u v}=c_{v u}=5$ would hold, contradicting the assumption $c_{u v}<c_{v u}$. Hence in (g) we have $\mu(u) \leq w_{2}<w^{\prime} \leq \mu(v)$ or $\mu(u) \leq\left(w_{2}+w_{3}\right) / 2<\left(w^{\prime}+w_{3}\right) / 2 \leq \mu(v)$.

\section{References}

1. G. Di Battista, P. Eades, R. Tamassia and I. G. Tollis, Graph Drawing: Algorithms for Visualization of Graphs, Prentice-Hall, Englewood Cliffs, NJ, 1999.

2. V. Dujmović, H. Fernau and M. Kaufmann, Fixed parameter algorithms for one-sided crossing minimization revisited, in Graph Drawing 2003, pp. 332-344, Lecture Notes in Computer Science, Vol. 2912, Springer-Verlag, Berlin, 2004.

3. V. Dujmović and S. Whitesides, An efficient fixed parameter tractable algorithm for 1-sided crossing minimization, in Graph Drawing 2002, pp. 118-129, Lecture Notes in Computer Science, Vol. 2528, Springer-Verlag, Berlin, 2002.

4. P. Eades and N. C. Wormald, Edge crossing in drawing bipartite graphs, Algorithmica 11 (1994), 379-403.

5. M. R. Garey and D. S. Johnson, Crossing number is NP-complete, SIAM J. Algebraic Discrete Methods 4 (1983), 312-316.

6. F. Harary and A. J. Schwenk, Trees with Hamiltonian square, Mathematika 18 (1971), 138-140.

7. F. Harary and A. J. Schwenk, A new crossing number for bipartite graphs, Util. Math. 1 (1972), 203-209.

8. M. Jünger and P. Mutzel, 2-Layer straight line crossing minimization: performance of exact and heuristic algorithms, J. Graph Algorithms Appl. 1 (1997), 1-25.

9. X. Y- Li and M. F. Sallmann, New bounds on the barycenter heuristic for bipartite drawing, Inform. Process. Lett. 82 (2002), 293-298. 
10. E. Mäkinen, Experiments on drawing 2-level hierarchical graphs, Internat. J. Comput. Math. 36 (1990), $175-181$.

11. X. Muñoz, W. Unger and I. Vrt'o, One-sided crossing minimization is NP-hard for sparse graphs, in Graph Drawing 2001, pp. 115-123, Lecture Notes in Computer Science, Vol. 2265, Springer-Verlag, Berlin, 2002.

12. H. Nagamochi, On the one-sided crossing minimization in a bipartite graph with large degrees, Theoret. Comput. Sci. 332 (2005), 417-446.

13. H. Nagamochi and N. Yamada, Counting edge crossings in a 2-layered drawing, Inform. Process. Lett. 91 (2004), 221-225.

14. C. Sechen, VLSI Placement and Global Routing Using Simulated Annealing, Kluwer, Dordrecht, 1988.

15. K. Sugiyama, S. Tagawa and M. Toda, Methods for visual understanding of hierarchical system structures, IEEE Trans. Systems Man Cybernet. 11 (1981), 109-125.

16. A. Yamaguchi and A. Sugimoto, An approximation algorithm for the two-layered graph drawing problem, in Proc. COCOON99, pp. 81-91, Lecture Notes in Computer Science, Vol. 1627, Springer-Verlag, Berlin, 1999.

Received September 17, 2003, and in revised form November 25, 2004. Online publication March 18, 2005. 